\title{
Wheat Thioredoxin (TaTrxh1) Associates With RD19-Like Cysteine Protease TaCP1 to Defend Against Stripe Rust Fungus Through Modulation of Programmed Cell Death
}

\author{
Beibei Shi, ${ }^{1}$ Xinbei Zhao, ${ }^{1,2}$ Min Li, ${ }^{1}$ Zihui Dong, ${ }^{1}$ Qichao Yang, ${ }^{1}$ Yang Wang, ${ }^{1}$ Haifeng Gao,,${ }^{3,+}$ \\ Brad Day ${ }^{4,5,+}$ and Qing $\mathrm{Ma}^{1,+}$ \\ ${ }^{1}$ State Key Laboratory of Crop Stress Biology for Arid Areas and College of Plant Protection, Northwest A\&F University, \\ Yangling, Shaanxi 712100, China \\ ${ }^{2}$ Institute of Plant Protection, Henan Academy of Agricultural Sciences/Key Laboratory of IPM of Pests on Crop (Southern North \\ China), Ministry of Agriculture, Key Laboratory of Crop Pest Control of Henan, Zhengzhou, Henan 450002, China \\ ${ }^{3}$ Institute of Plant Protection, Xinjiang Academy of Agricultural Sciences/Key Laboratory of Integrated Pest Management on \\ Crop in Northwestern Oasis, Ministry of Agriculture and Rural Affairs, Urumqi, Xinjiang 830091, China \\ ${ }^{4}$ Department of Plant, Soil and Microbial Sciences, Michigan State University, East Lansing, MI, U.S.A. \\ ${ }^{5}$ Plant Resilience Institute, Michigan State University, East Lansing, MI, U.S.A.
}

Accepted 8 December 2020.

Thioredoxins (Trxs) function within the antioxidant network through modulation of one or more redox reactions involved in oxidative-stress signaling. Given their function in regulating cellular redox, Trx proteins also fulfill key roles in plant immune signaling. Here, TaTrxh1, encoding a subgroup $h$ member of the Trx family, was identified and cloned in wheat (Triticum aestivum), which was rapidly induced by Puccinia striiformis f. sp. tritici invasion and salicylic acid (SA) treatment. Overexpression of TaTrxh1 in tobacco (Nicotiana benthamiana) induced programmed cell death. Silencing of TaTrxh1 in wheat enhanced susceptibility to $P$. striiformis $\mathbf{f}$. sp. tritici in different aspects, including reactive oxygen species accumulation and pathogenresponsive or -related gene expression. Herein, we observed that the cellular concentration of SA was significantly reduced in TaTrxh1-silenced plants, indicating that TaTrxh1 possibly regulates wheat resistance to stripe rust through a SA-associated defense signaling pathway. Using a yeast two-hybrid screen to identify TaTrxh1-interacting partners, we further show that interaction with TaCP1 (a RD19-like cysteine protease) and subsequent silencing of $\mathrm{TaCPI}$ reduced wheat resistance to $P$. striiformis f. sp. tritici. In total, the data presented herein demonstrate that TaTrxh1 enhances wheat resistance against

B. Shi and X. Zhao contributed equally to this work.

${ }^{\dagger}$ Corresponding authors: H. Gao; ghf20044666@163.com,

B. Day; bday@msu.edu, and Q. Ma; maqing@nwsuaf.edu.cn

Funding: This research was supported by the National Natural Science Foundation of China (grant numbers U1903110 and 31571960) and the 111 Project from the Ministry of Education of the People's Republic of China (grant number B07049) to Q. Ma. Research in the laboratory of B. Day was supported by the United States National Science Foundation Plant-Biotic Interactions Program (IOS-1557437) and the National Institute of General Medical Sciences (1R01GM125743).

*The $\boldsymbol{e}$-Xtra logo stands for "electronic extra" and indicates there is supplementary material published online.

The author(s) declare no conflict of interest.

(c) (1) \&) Copyright $(92021$ The Author(s). This is an open access article distributed under the CC BY-NC-ND 4.0 International license.
P. striiformis f. sp. tritici via SA-dependent resistance signaling and that TaTrxh1 interaction with TaCP1 is required for wheat resistance to stripe rust.

Keywords: thioredoxin, cysteine protease, wheat stripe rust, programmed cell death, salicylic acid

Thioredoxins (Trxs) are present in all organisms and contain a highly conserved amino acid motif, Cys-Gly-Pro-Cys (Gelhaye et al. 2005; Meyer et al. 2012), which has been demonstrated to be necessary and required for redox signaling. Specifically, this class of proteins functions through modifying a covalent mixed disulfide bond that exists in oxidized forms (i.e., Trx- $\mathrm{S}_{2}$ ) and dithiol [i.e., TRX-(SH) $)_{2}$ (Jacquot et al. 2002; Meyer et al. 2008). To date, at least 20 Trx genes have been reported in Arabidopsis thaliana, which are classified into six subfamilies ( $f, h, m, o, x$, and $y$ ) (Meyer et al. 2002, 2012). Members of subfamilies $f, m, x$, and $y$ and were localized to the chloroplast (Meyer et al. 2008, 2009), while members of subfamilies $h$ and $o$ showed localization patterns including the mitochondria, cytoplasm, and nuclei (Gelhaye et al. 2005; Marchal et al. 2014; Meyer et al. 2008).

Given their highly ubiquitous and diverse cellular and tissue patterns of expression, it is reasonable to surmise that Trxs participate in numerous physiological processes in plants. For example, Trxs have demonstrable roles in seed development, wherein they function in the reduction of endosperm storage proteins, altering their sensitivity to hydrolysis (Thormählen et al. 2017; Wang et al. 2017; Zhou et al. 2013). Additionally, previous research has also shown that $\operatorname{Trxm} 3$, which is localized in the chloroplast, is required for processes associated with root growth and leaf development (Reichheld et al. 2010). As a function of stress response processes, Trxs have been shown to be required for robust signaling during abiotic stress response, including drought (Sahrawy et al. 1996), extreme temperatures (Park et al. 2009; Xie et al. 2009), and $\mathrm{H}_{2} \mathrm{O}_{2}$ perception (Spector et al. 1988).

Owing to their function in regulating cellular redox, Trxs also play a diverse role in the regulation of plant immune signaling (Mata-Pérez and Spoel 2019). For example, Ralstonia 
solanacearum utilizes host Trxs to activate the glutathione degrading activity of RipAY (Mukaihara et al. 2016). This reaction results in the inhibition of pathogen-associated molecular pattern-triggered immunity (PTI) recognized by pattern recognition receptors that comprises basal level of disease resistance in a host (Dodds and Rathjen 2010; Jones and Dangl 2006). Moreover, Trx- $z$ interacts with Cf-9, a tomato leucine-rich repeat resistance protein, which is a positive regulator of Cf-9-mediated hypersensitive response (HR) response in tomato (Jones and Dangl 2006; Macho and Zipfel 2015; Rivas et al. 2004).

The $h$-type Trx proteins represent the largest Trx family and are a signaling intermediate of antioxidant activities (Bashandy et al. 2010; Sun et al. 2010). For example, OsTRXh1 regulates reactive oxygen species (ROS) accumulation in the apoplast and influences plant development and salt-stress responses (Zhang et al. 2011), while CaTrxhl plays a key role in the activation of HR-associated defense responses in pepper (Yi et al. 2007). In addition, an $h$-type Trx in tobacco NtTRXh3 is involved in the resistance of tobacco to virus infection and abiotic oxidative stress (Sun et al. 2010). By regulating the conformational state of NPR1 through the reduction of the NPR1 (nonexpression of PR1) disulfide bond, AtTrxh5 can promote the expression of defense-related genes induced by salicylic acid (SA) (Kneeshaw et al. 2014; Yun et al. 2016). Moreover, the expression of AtTrxh5 is highly induced following the perception of pathogen elicitors, wounding, abscission, and senescence (Laloi et al. 2004; Reichheld et al. 2002; Tada et al. 2008). While several recent studies have provided evidence of a role for Trxs in pathogen resistance, the role of Trxs in conferring pathogen resistance in wheat has not been demonstrated, nor has it been shown that these proteins play a role in programmed cell death (PCD).

Wheat stripe rust, caused by Puccinia striiformis f. sp. tritici, is responsible for devastating yield reductions in wheat (Triticum aestivum). As an obligate biotrophic basidiomycete, $P$. striiformis f. sp. tritici produces urediospores on the leaves of susceptible wheat cultivars, ultimately leading to pathogen feeding and a resultant yield reduction (Singh et al. 2016). In response to $P$. striiformis $\mathrm{f}$. sp. tritici infection, resistant wheat cultivars activate PCD-associated processes, resulting in the elicitation of tissue necrosis, a process hypothesized to restrict the invasion of $P$. striiformis f. sp. tritici (Chen et al. 2014). While emerging data has illuminated our understanding of the various processes that are activated in response to infection, additional studies describing the function of both pathogenderived effectors and host-resistance factors are key in advancing our understanding of this complex host-pathogen interaction. Indeed, such approaches have been valuable in guiding breeding approaches for the development of new disease resistant cultivars (Eichmann and Hückelhoven 2008). From these, several key genes required for disease resistance signaling in response to $P$. striiformis f. sp. tritici have been identified, including the transcription factor TabZIPl (Zhang et al. 2008), TaHSP70 (Duan et al. 2011), a metacaspase gene TaMCA4 (Wang et al. 2012a), TaNPSN genes (Wang et al. 2014), and calcineurin B-like interacting protein kinase $\mathrm{TaC}$ IPK10 (Liu et al. 2019).

Here, we describe the cloning of an $h$-type Trx, TaTrxh1, and its involvement in resistance to $P$. striiformis $\mathrm{f}$. $\mathrm{sp}$. tritici in wheat. In total, we demonstrate that overexpression of TaTrxh1 induces cell death and ROS accumulation. Using a combination of in vivo-based interaction approaches, we demonstrate that a cysteine protease (TaCP1) is a downstream signaling protein of TaTrxh1. In total, the current work described herein provides evidence supporting a link between TaTrxh1 and TaCP1 and the regulation of cell death following infection of wheat by $P$. striiformis f. sp. tritici.

\section{RESULTS}

Sequence analysis of wheat $h$-type Trx.

Based on publicly available sequence data (GenBank accession number AY072771.1), DNA primers (Supplementary Table S1) were designed for PCR-based cloning of a wheat (Triticum aestivum) $h$-type Trx. Using cDNA prepared from T. aestivum cv. Suwon11 (Su11), a 357-bp DNA sequence was obtained. This coding sequencing (CDS) encode a 118-amino acid protein with an estimated molecular mass of $12.69 \mathrm{kDa}$ and an isoelectric point of 5.29. Blast analysis of the CDS of TaTrxh in URGI revealed that three copies of TaTrxh exist in T. aestivum cv. Chinese Spring, with 99, 95, and 94\% nucleotide sequence identity, which were located on chromosomes 2A, 2B, and 2D, respectively (Supplementary Fig. S1A). The acquired TaTrxh clones (GenBank accession number AY072771.1) as well as four additional $h$-type Trx genes from T. aestivum (GenBank accession number AF438359.1, AF286593.2, EU706448.1, and AJ009762.1) were analyzed by multiple DNA and mitochondrial RNA (mRNA) sequence alignment approaches (Supplementary Fig. S1B and C). Using this approach, we observed that the acquired TaTrxh (GenBank accession number AY072771.1) had the closest relationship (i.e., sequence-based similarity) with HvTrxh (AY245454.1), with the next closest alignment being to OsTrxhl (Os07g0186000). Based on this, the protein was named wheat Trxh1 (TaTrxh1) (Supplementary Fig. S1D).

\section{TaTrxh1 is significantly induced}

by $P$. striiformis f. sp. tritici infection and SA treatment.

Trxs are involved in plant disease resistance signaling (Mukaihara et al. 2016). To analyze the transcriptional profiles (i.e., mRNA accumulation) of wheat resistance to stripe rust, accumulation of five TaTrxh mRNAs was evaluated in leaf samples collected at $0,12,24,36,48,72,96$, and $120 \mathrm{~h}$ postinoculation (hpi) with $P$. striiformis f. sp. tritici. As shown in Figure 1A, TaTrxh1 expression was significantly induced at 96 and 120 hpi during an incompatible interaction, with a 3.3- and 5.3-fold increase, respectively, when compared with samples collected at 0 hpi. Interestingly, we did not observe a significant difference in TaTrxh1 mRNA accumulation during a compatible interaction, indicating that TaTrxhl may regulate wheat resistance to stripe rust.

Plant hormones play an important role in numerous signal transduction processes. As a link to the current study, the activity of Trxs has been described has a key component of SAmediated immune signal transduction cascades, suggesting a link between redox-based processes and hormone signaling in response to pathogen infection (Mata-Pérez and Spoel 2019). To investigate the role of TaTrxhl in immune signaling and to further determine the response of TaTrxhl following hormone perception, we treated wheat leaves with SA and methyljasmonate (MeJA) and evaluated the mRNA accumulation of TaTrxh1. As shown in Figure 1B, following treatment of plants with SA, the accumulation of TaTrxh1 mRNA was significantly increased at 3 and $6 \mathrm{~h}$ posttreatment (hpt), with an observed 2.6- and 4.1-fold increase, respectively. TaTrxhl mRNA accumulation did not change significantly (in comparison with untreated samples) following MeJA treatment. Taken together, TaTrxhl could enhance the resistance of wheat to P. striiformis f. sp. tritici through SA-mediated signaling.

\section{Overexpression of TaTrxh1 in tobacco leaves can induce the cell death.}

To further understand the mechanism or mechanisms underpinning the role of TaTrxhl in defense signaling in wheat following $P$. striiformis $\mathrm{f}$. sp. tritici infection, the full CDS of 
TaTrxhl was cloned into the heterologous expression vector PVX106 and was expressed in tobacco (Nicotiana benthamiana) leaves using Agrobacterium tumefaciens. Our aim was to evaluate the overexpression cell-death activity of TaTrxhl and its ability to suppress PCD. To do this, we exploited the activity of BAX, a death promoting member of the Bcl-2 family of proteins that can trigger cell death, which has similar physiological characteristics to plant HRs when expressed in plants (Lacomme and Santa Cruz 1999). As shown in Figure 2, PVX106:TaTrxh1+Bax, PVX106+Bax, and PVX106:TaTrxh1 expression zones showed the induction of necrosis, while no celldeath phenotype was observed in regions infiltrated with PVX106 alone or buffer-only controls.

\section{Suppression of TaTrxh1 reduces wheat resistance to $P$. striiformis f. sp. tritici.}

Virus-induced gene silencing (VIGS) is a common approach tool for the evaluation of the function and activity of plant disease resistance genes (Bennypaul et al. 2012). Based on the observation that the expression of TaTrxhl increased when wheat was challenged with $P$. striiformis f. sp. tritici, we
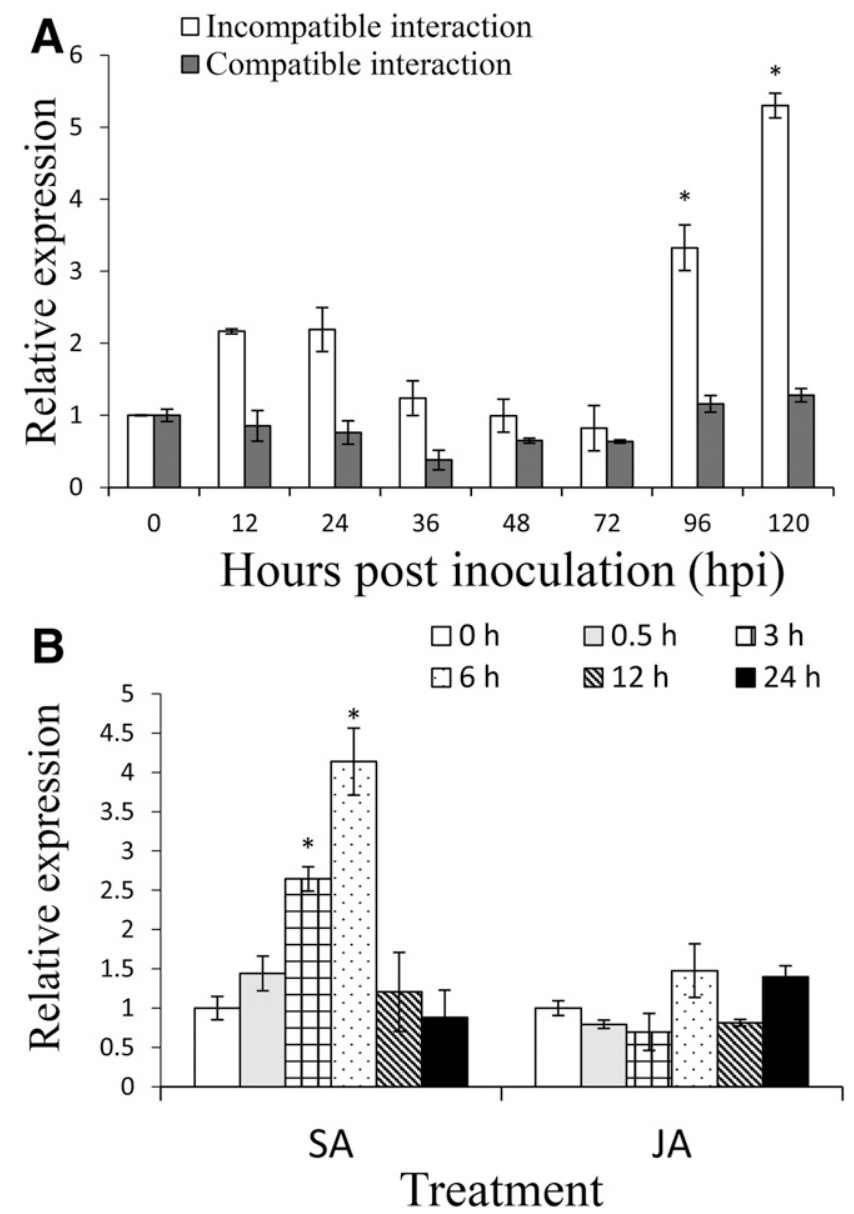

Fig. 1. Expression time course of TaTrxhl in wheat leaves in response to Puccinia striiformis f. sp. tritici infection and salicylic acid (SA) treatment. A, The accumulation of TaTrxh1 mRNA after P. striformis f. sp. tritici infection. The Su11 leaves inoculated with $P$. striiformis f. sp. tritici race CYR23 (incompatible interaction) and race CYR31 (compatible interaction). B, SA treatment enhances TaTrxh1 expression. Wheat seedlings were treated with $100 \mathrm{mM}$ methyl jasmonate (MeJA) and $2 \mathrm{mM} \mathrm{SA}$. The asterisk $(*)$ indicates a statistically significant difference $(P<0.05)$, using Student's $t$ test, while $0 \mathrm{~h}$ was normalized as 1 , and there were three independent biological replicates. All data were determined by real-time quantitative PCR with wheat TaEF-1a as a reference gene. Wheat leaves treated with distilled water were included as mock control. utilized VIGS to silence TaTrxh1 in wheat, to evaluate its inplanta contribution in response to $P$. striiformis f. sp. tritici infection. As shown in Figure 3A, expression of BSMV: $\gamma-T a P D S$ (BSMV vector carrying a segment from wheat phytoene desaturase gene) was used as a positive control and induced a signature photobleaching response on inoculated plants (Wang et al. 2012a and b). Plants inoculated with BSMV (BSMV: $\gamma /$ BSMV: $\gamma$-TaTrxh1) yielded mild chlorotic mosaic symptoms, indicating the efficacy of gene silencing. As shown, obvious mild chlorotic mosaic symptoms were observed on the fourth leaves in BSMV: $\gamma$ and BSMV: $\gamma$-TaTrxh1 plants at 10 days postinoculation (dpi), while leaves inoculated with BSMV: $\gamma-T a P D S$ displayed signs of photobleaching (Fig. 3A). Next, to evaluate the role of BSMV: $\gamma$-TaTrxhl in wheat resistance to stripe rust, the silencing efficiency of TaTrxhl-infected plants was tested using real-time quantitative PCR (RTqPCR) assay at 24,48 , and 120 hpi with avirulent $P$. striiformis $\mathrm{f}$. sp. tritici CYR23 and virulent $P$. striiformis f. sp. tritici CYR31 on the fourth leaves of inoculated wheat. As shown in Figure $3 \mathrm{C}$ and D, the accumulation of TaTrxh1 was reduced approximately 70 to 90\% compared with BSMV: $\gamma$ control plants, suggesting that TaTrxhl was silenced using the BSMV system.

To further investigate this mechanism, we evaluated the induction of cell-death phenotypes in CYR23-inoculated BSMV: $\gamma$, BSMV: $\gamma$-TaTrxhl and mock-inoculated plants at 15 dpi. As shown, the necrotic area of BSMV: $\gamma$-TaTrxh1 plants was more pronounced than in control plants, with a reduced number of uredia observed on BSMV: $\gamma$-TaTrxhl plants (Fig. 3B). As shown, all leaves showed considerably pronounced uredia phenotypes after inoculating with $P$. striiformis f. sp. tritici CYR31, while the TaTrxh1-silenced plants produced more uredia. As a final step to investigate the host response in TaTrxh1-silenced plants after $P$. striiformis f. sp. tritici infection, two pathogenesis-related $(P R)$ genes, TaPRI and $T a P R 3$, were evaluated using RT-qPCR. As shown in Figure 3E, both mRNAs were significantly reduced compared with control plants. Based on the sum of this series of data, we conclude that TaTrxh1 plays a positive-regulatory role in resistance signaling to stripe rust.

\section{TaTrxh1 silencing decreases ROS accumulation.}

To further define pathogen-induced defense changes as a function of TaTrxh1 activity following P. striiformis f. sp. tritici infection, we next evaluated the production of $\mathrm{H}_{2} \mathrm{O}_{2}$ following $P$. striiformis f. sp. tritici infection. As a point of reference, the formation of a substomatal vesicle was considered a successful infection site. As shown in Figure 4A and $\mathrm{B}$, the area of $\mathrm{H}_{2} \mathrm{O}_{2}$ generation was significantly reduced at $120 \mathrm{~h}$ in TaTrxhlsilenced plants after inoculating with CYR23, as compared with control (i.e., unsilenced) plants. Concomitant with this, and not unsurprisingly, the expression of TaCAT (catalase) and TaSOD (superoxide dismutase), both of which are involved in ROS signaling, were induced (Fig. 4C). These data support the hypothesis that the function of TaTrxhl in wheat defense against P. striiformis f. sp. tritici is ROS-dependent. As an additional measure of the role of TaTrxh1 in wheat defense against P. striiformis f. sp. tritici, we also evaluated the histology of P. striiformis f. sp. tritici race CYR23-infected wheat. Using this approach, we observed that hyphal length and the leaf area of pathogen colonization were significantly increased in silenced plants (Fig. 4D to $\mathrm{H}$ ), further confirming that TaTrxhl is required for robust resistance to $P$. striiformis $\mathrm{f}$. sp. tritici.

Effects of TaTrxh1 silencing on SA and JA accumulation.

The SA- and JA-dependent signaling pathways are two important disease resistance-signaling pathways in plants. As 
shown above, we observed that TaTrxh1 mRNA accumulation was induced following exogenous application of SA (Fig. 1B). We also detected that the expression of TaPRI and TaRP3 were suppressed in TaTrxhl-silenced plants. TaPRI and TaRP3 are marker genes in the SA signaling pathway and can be induced by SA. Thus, we speculated that TaTrxhl was involved in SA signaling. To confirm our view, we collected samples of the control and TaTrxhl-silenced plants at 18 and $24 \mathrm{~h}$ after inoculating with $P$. striiformis f. sp. tritici race CYR23 to quantify SA and JA content. As shown in Figure 5, the concentration of SA was significantly reduced in TaTrxhl-silenced plants at 24 hpi, with an approximate $40 \%$ reduction in SA, as compared with control plants. Consistent with our observations above, we did not observe a significant change in the level of JA. Additionally, the SA biosynthesis pathway-related genes (i.e., TaICS1 [isochorismate synthase 1] and TaPAL [phenylalanine ammonia-lyase]) and SA-regulated gene TaNPR1 were evaluated using RT-qPCR. As shown in Supplementary Fig. S2, the expression of TaICS1 (120 hpi), TaPAL (24 and $120 \mathrm{hpi}$ ), and TaNPRl (48 and $120 \mathrm{hpi}$ ) were significantly reduced in TaTrxh1-silenced plants after inoculating with CYR23 compared with control plants, which further indicated that TaTrxhl is involved in SA signaling following $P$. striiformis f. sp. tritici infection. Taken together, these data support the hypothesis that TaTrxh1 regulates wheat resistance to stripe rust through the SA signaling pathway.

\section{TaTrxh1 interacts with TaCP1, a wheat RD19-like cysteine protease.}

As a means to investigate the mechanism underpinning the TaTrxhl-induced cell-death response in tobacco, we used TaTrxh1 as a bait protein to screen for interacting proteins, using the yeast two-hybrid system. Using this approach, we screened a wheat yeast two-hybrid for TaTrxh1-interacting proteins and, through this, developed a candidate list of approximately 40 TaTrxh1-interacting proteins (Supplementary Table S2). From this list, we focused on the characterization of a partial cDNA clone encoding cysteine proteinase 1 (CP1), which is a key enzyme that regulates PCD and plays roles in numerous aspects of cell signaling in plants (Van Hautegem et al. 2015). Using the sequence derived from these clones, we isolated the full-length CDS and found that it is predicted to encode a papain-like cysteine proteinase (PLCPs) (Richau et al. 2012). Evolutionary analysis showed that this cysteine proteinase had the closest relationship with RD19C from among 30 papain-like cysteine proteases found in Arabidopsis (Supplementary Fig. S3A). The derived TaCP1 clone was predicted to possess a conserved catalytic triad, Cys-His-Asn, with an additional two specific pairs of highly conserved cysteine (Cys) residues found in RD19A-like proteases from among four Arabidopsis RD19A-like proteins analyzed (Supplementary Fig. S3B).

To further evaluate the results of the yeast two-hybrid analysis, a full-length clone of $T a C P 1$ was cloned into the yeast expression vector pGADT7. TaTrxhl and TaCPl were cotransformed into yeast for further evaluation. As shown in Figure 6A, we observed that yeast containing both TaTrxh1 and TaCP1 grew on synthetic defined (SD)-Trp-Leu-His and SDTrp-Leu-His-Ade media; further confirmation of this interaction was obtained in combination with the development of blue staining in the presence of $\mathrm{X}$ - $\alpha$-galactosidase (X- $\alpha-\mathrm{Gal})$. The negative control group showed no significant growth. The interaction strength of TaTrxh4 and TaCP1 was further confirmed by spotting yeast dilutions (Fig. 6A). Lastly, biomolecular fluorescence complementation (BiFC) was used to confirm the interaction between TaTrxh1 and TaCP1 in plant cells, wherein pSPYCE::TaTrxh1 and $p S P Y N E:: T a C P 1$ vector were transformed in A. tumefaciens for expression in $N$. benthamiana. As shown in Figure 6B, only the TaTrxh1-cYFP/nYFP$\mathrm{TaCP} 1$ showed a strong yellow fluorescence signal, indicating that TaTrxh1 interacts with TaCP1 in planta.

\section{TaCP1 positively regulates wheat resistance to $P$. striiformis f. sp. tritici.}

Wang et al. (2012a) previously reported that TaMCA4, a wheat metacaspase, is induced by $P$. striiformis f. sp. tritici and functions in PCD development, demonstrating the importance of cysteine proteinases in wheat immunity to pathogen infection. Similarly, Bernoux et al. (2008) showed that Arabidopsis RD19-mediated resistance to Ralstonia solanacearum is conferred by the interaction with the $\mathrm{R}$ protein PopP2. To evaluate the activity of $R D 19$ in wheat immunity to $P$. striiformis f. sp. tritici, we quantified the mRNA accumulation of $T a C P l$ accumulation following inoculation with P. striiformis f. sp. tritici races CYR23 and CYR31. As shown in Figure 7A, we observed that TaCP1 mRNA accumulation was induced following $P$. striiformis f. sp. tritici infection. Unsurprisingly, and consistent with our observations, described above, we also observed that the expression of TaCP1 mRNA was significantly different in both compatible and incompatible interactions, with expression in the incompatible interaction being 14.0- and 2.6-fold higher compared with the compatible interaction at 12 and $96 \mathrm{hpi}$, respectively.

Using the BSMV-VIGS system, we demonstrate the TaCPI is required for resistance to $P$. striiformis f. sp. tritici, using a 230-bp segment of TaCPl inserted into BSMV: $\gamma$. After
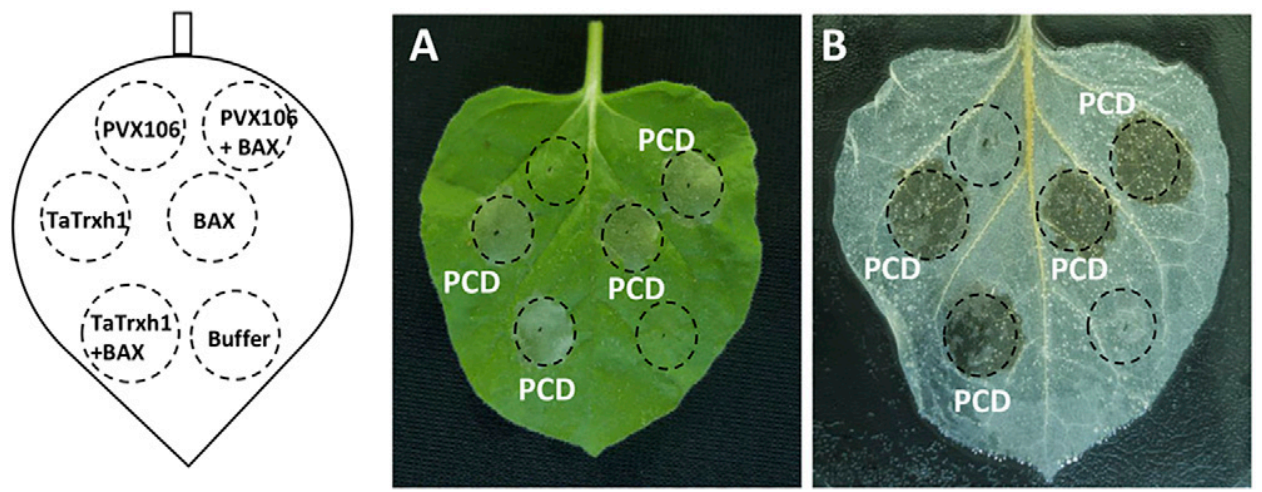

Fig. 2. Overexpression of TaTrxh1 in tobacco (Nicotiana benthamiana) leads to cell death. A, Tobacco leaves were infiltrated with Agrobacterium tumefaciens. After 24 h, Bax was infiltrated at PVX106:TaTrxh1+Bax and PVX106+Bax sites. Symptoms on leaves of N. benthamiana were assessed 5 days after infiltration. B, The same leaves were placed in $100 \%$ ethanol for decolorizing. 
successful virus inoculation, mild chlorotic mosaic symptoms and photobleaching were observed on BSMV: $\gamma$-as and BSMV: $\gamma-T a P D S$, respectively. At 10 dpi (Fig. 7B), urediospores of $P$. striiformis $\mathrm{f}$. sp. tritici race CYR23 (avirulent) were inoculated on the fourth leaves of BSMV: $\gamma$-as, which all had cell-death phenotypes, with the necrotic area of BSMV: $\gamma-T a C P 1$ being larger than negative and blank controls, and a small amount of uredia were produced at $15 \mathrm{dpi}$ (Fig. 7C). The silencing efficiency of $\mathrm{TaCPl}$ was approximately 78 to $86 \%$ (Fig. 7D).

To further understand how TaCP1 participates in wheat resistance, a histological examination of $P$. striiformis $\mathrm{f}$. sp. tritici growth was performed in both control and silenced plants (Supplementary Fig. S4) by analyzing the number of hyphal branches, the length of hyphae, and the area of pathogen colonization and the results showed all were increased significantly in silenced plants compared with control plants at $120 \mathrm{hpi}$ (Fig. 7E to G). The expression of TaPRI and TaPR3 were all reduced significantly at 24 to 120 hpi in BSMV: $\gamma-T a C P 1$ plants when challenged with the avirulent race CYR23 (Fig. $7 \mathrm{H}$ ). In addition, the expression of TaSOD and TaCAT were significantly induced at 120 and $48 \mathrm{hpi}$, respectively (Fig. 7I). Therefore, TaCPl was a positive regular in wheat resistance.

\section{DISCUSSION}

The modulation of plant immune response signaling processes is intimately associated with the rapid production and clearing
A Control

BSMV: BSMV: BSMV:

Mock TaPDS $\gamma$
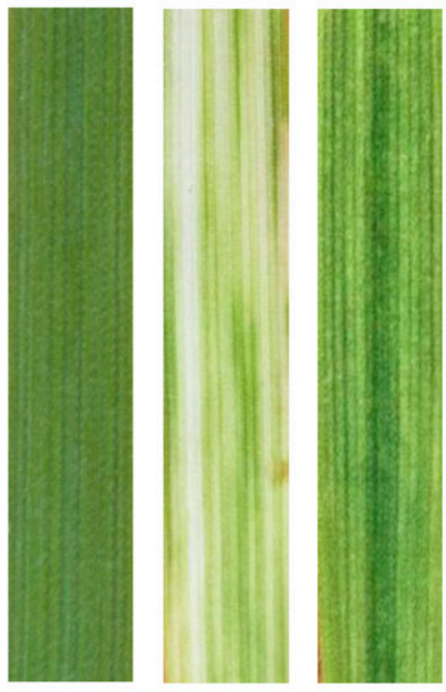

C

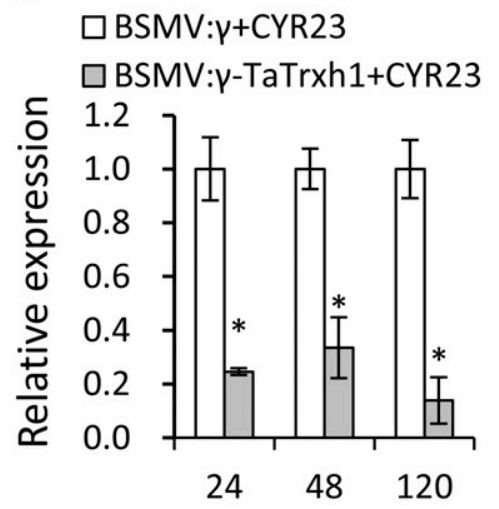

Hours post-inoculation (hpi)
B TaTrxh1

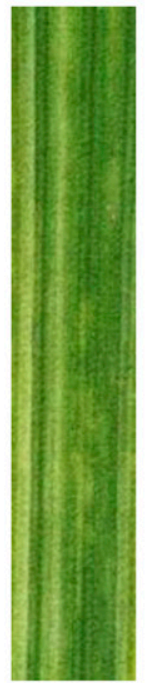

B

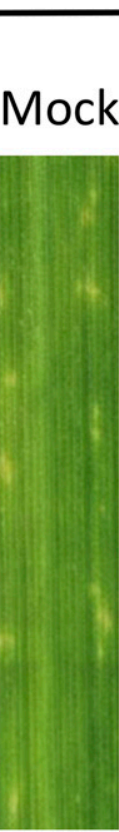

CYR23

BSMV: BSMV:

$\checkmark$

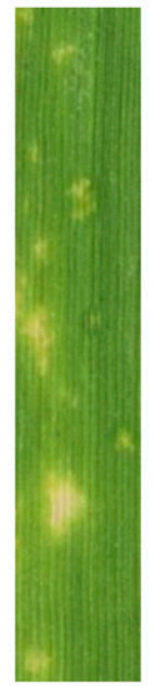

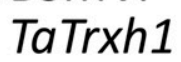

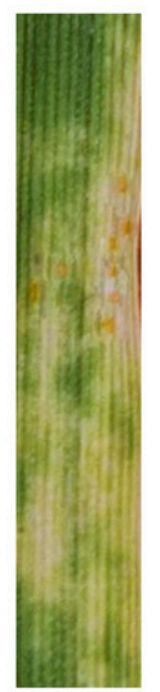

CYR31

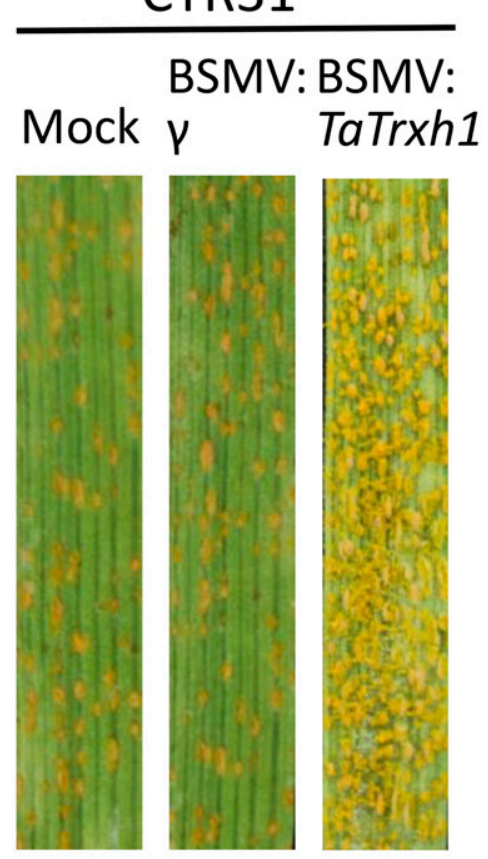

D

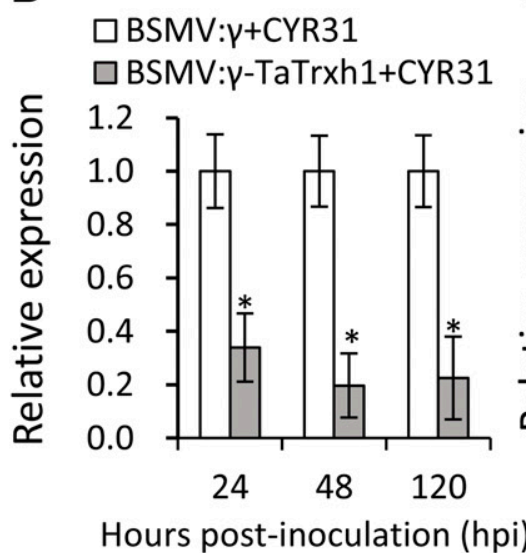

E
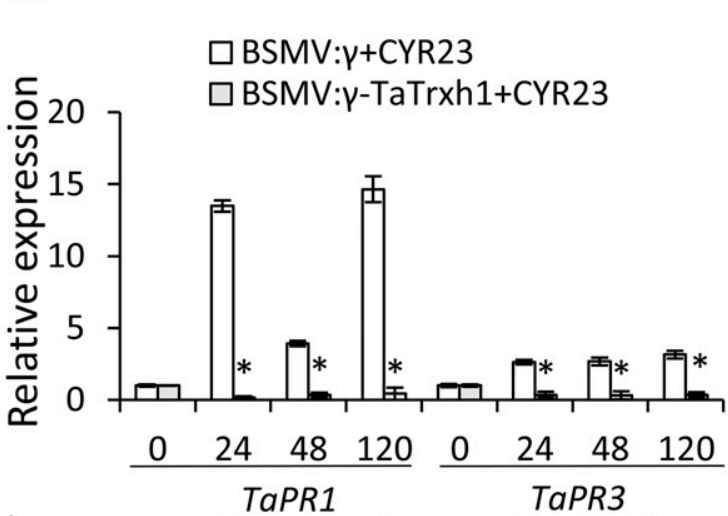

Hours post-inoculation (hpi)

Fig. 3. Suppression of TaTrxh1 reduces wheat resistance to Puccinia striiformis f. sp. tritici, as indicated by the barley stripe mosaic virus (BSMV)-induced gene silencing. A, Photo-bleaching and mild chlorotic mosaic symptoms were observed on the fourth leaves of BSMV: $\gamma$ - TaPDS, BSMV: $\gamma$, and BSMV: $\gamma$-TaTrxh1, respectively, at 10 days postinoculation (dpi), while there were no symptoms on the mock leaves treated with FES buffer. B, Fourth leaves preinoculated with BSMV: $\gamma$ and BSMV: $\gamma$-TaTrxh1 were challenged with urediospores of P. striiformis f. sp. tritici race CYR23 or CYR31. Typical leaves were photographed at $15 \mathrm{dpi}$. C, and D, The relative expression of TaTrxh1 was decreased in TaTrxh1-silenced plants inoculated with CYR23 and CYR31, respectively. E, The expressions of resistance-related genes in TaTrxh1 silenced plants were significantly reduced compared with BSMV: $\gamma$ after inoculation with $P$. striiformis f. sp. tritici race CYR23. TaPR1: pathogenesis-related protein (AJ007348.1), TaPR3: chitinase 3 (AB029936.1). $2^{-\triangle A C t}$ method was used and each sample had three independent biological repeats. Asterisks (*) indicate a significant difference $(P<0.05)$, using Student's $t$ test. TaEF-1a mRNA accumulation was monitored as a control. 
of reactive oxygen (Vieira Dos Santos and Rey 2006). As a key regulator of this process, Trxs function as critical components of this highly conserved defense mechanism and are required for the modulation of the redox status of components involved in pathways linked to oxidative-stress and gene expression regulation. Here, we present the identification and characterization of TaTrxh1, a positive regulator of biotic stress signaling in wheat. In total, the data described in the current study support
A

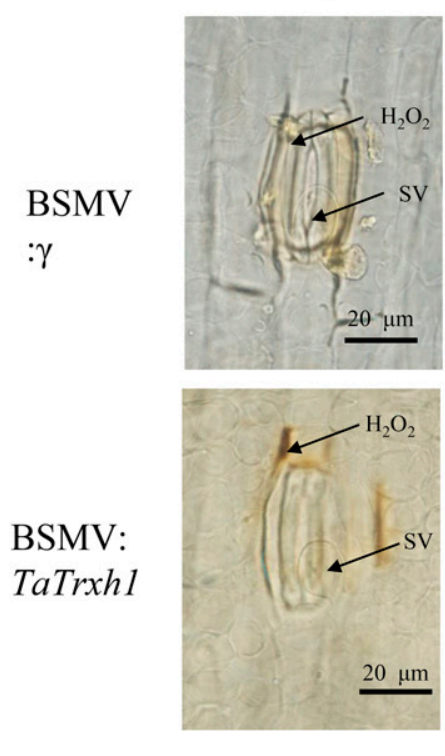

24 dpi

D
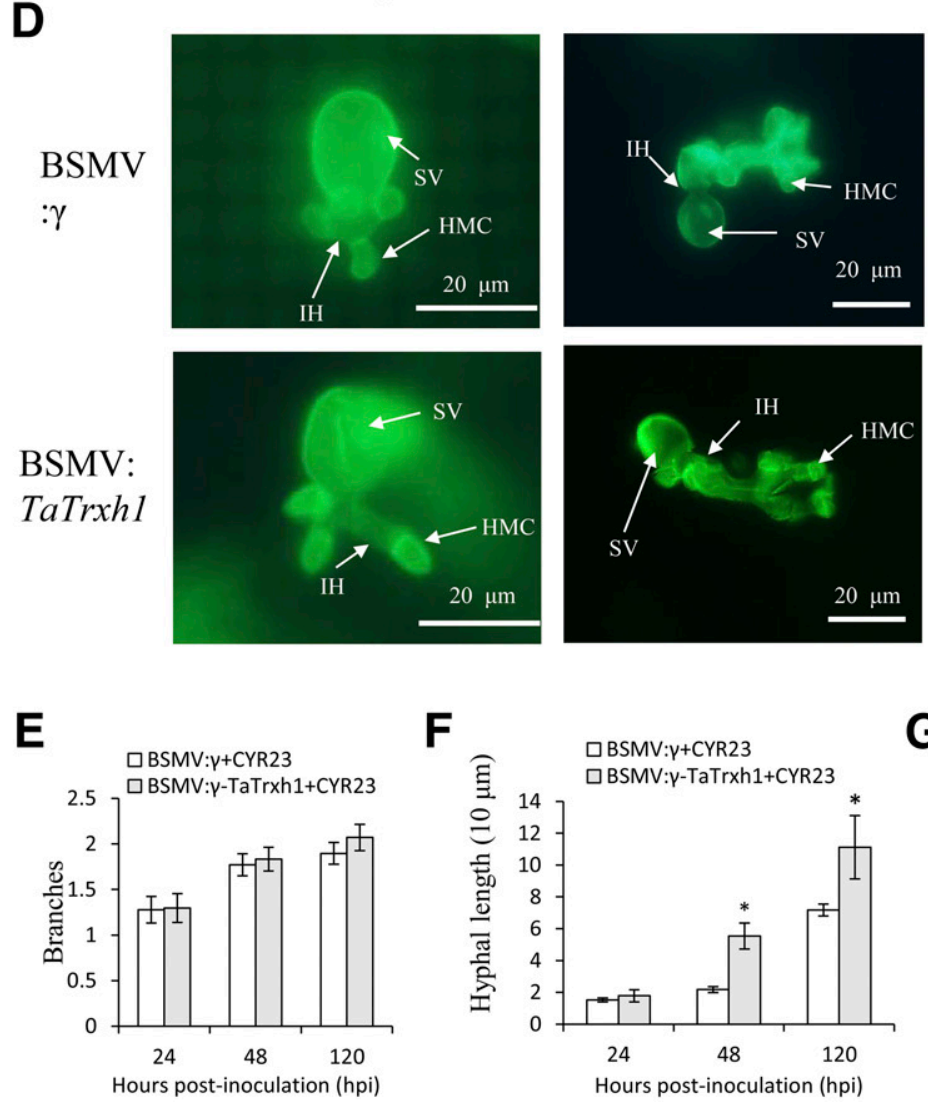

$\mathbf{F}$

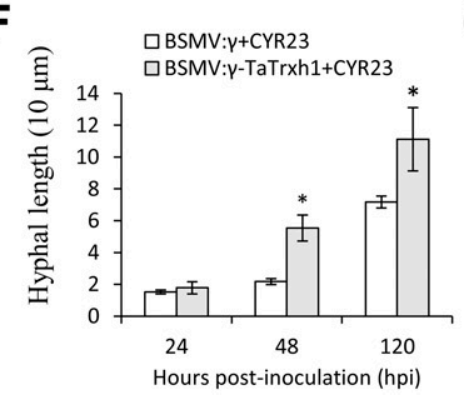

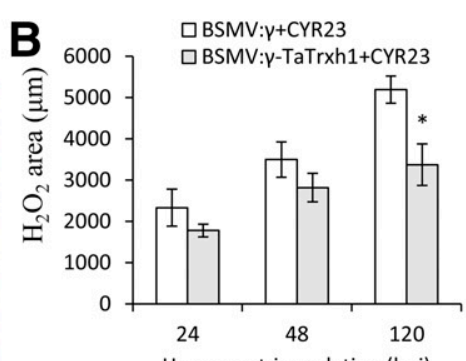

Hours post-inoculation (hpi)

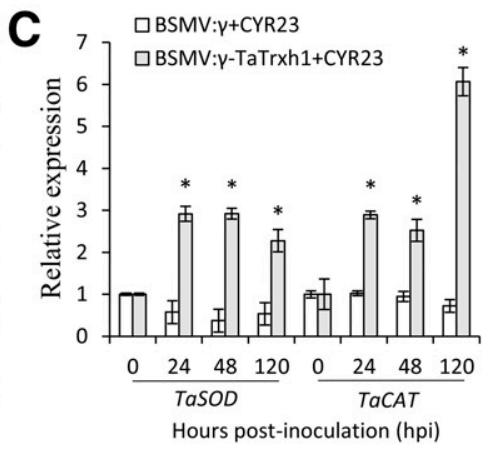

$120 \mathrm{dpi}$
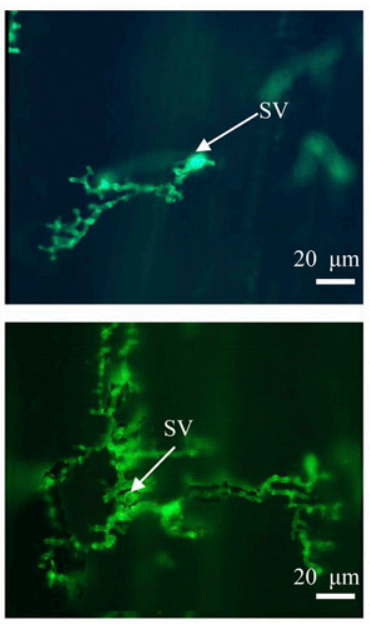

G

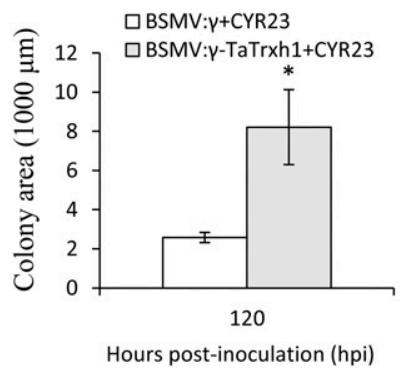

Fig. 4. Response of silenced plants after inoculation with the stripe rust fungus. $\mathbf{A}, \mathrm{H}_{2} \mathrm{O}_{2}$ accumulation observations at infection sites pretreated with $\mathrm{BSMV} / \gamma$ and BSMV: $\gamma$-TaTrxh1 and infected with CYR23. B, The area of $\mathrm{H}_{2} \mathrm{O}_{2}$ stained by 3,3'-diaminobenzidine was measured. C, TaSOD and TaCAT mRNA accumulations were significantly induced in silenced plants. D, Expansion of stripe rust race CYR23 as observed in TaTrxh1-silenced plants. E, Hyphal branch number, $\mathbf{F}$, hyphal length, and $\mathbf{G}$, colony area averages were calculated. Averages were calculated from 50 infection sites wherein the formation of a substomatal vesicle was observed, which is a marker for the induction of success fungal penetration. Asterisks $(*)$ indicate significant difference $(P<0.05)$ from BSMV: $\gamma$, using Student's $t$ tests. HMC $=$ haustorial mother cell, $\mathrm{IH}=$ infection hypha, and SV = substomatal vesicle. Bar $=20 \mu \mathrm{m}$. 
a role for TaTrxh1 as a SA-dependent regulator of $P$. striiformis f. sp. tritici-induced defense signaling in wheat and, in combination with $\mathrm{TaCP} 1$, is required for robust immune signaling activation.

Previous work demonstrated that Trxs are required for both PTI and effector-triggered immunity (Arsova et al. 2010; Mukaihara et al. 2016; Rivas et al. 2004). Indeed, each of these studies, including additional work describing the expression of AtTrxh5 in Arabidopsis (Reichheld et al. 2002), CaTrxh1 in pepper (Yi et al. 2007), NtTRXh3 in tobacco (Sun et al. 2010), and $O s T R X h 1$ in rice (Zhang et al. 2011) correlated the induced mRNA accumulation of Trx with pathogen perception and the subsequent activation of robust immune signaling processes.

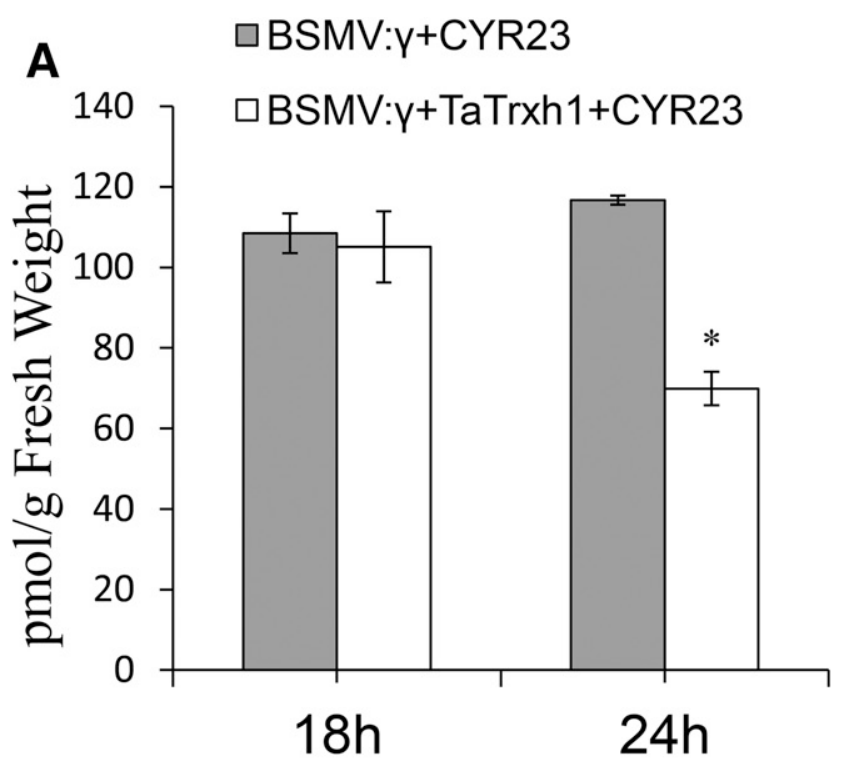

B 口BSMV: $\gamma+C Y R 23$

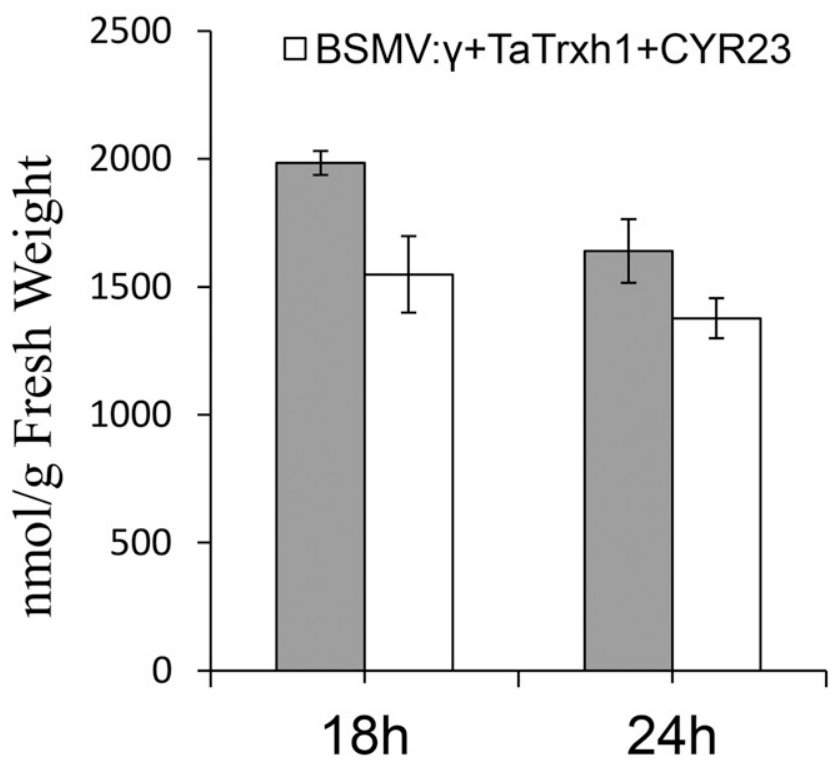

Fig. 5. Quantification of salicylic acid (SA) and jasmonic acid (JA) in Puccinia striiformis f. sp. tritici race CYR23-inoculated TaTrxh1-silenced plants. A, SA accumulation was significantly reduced. B, Accumulation of jasmonic acid (JA) remained unchanged at 18 and $24 \mathrm{~h}$ postinoculation. Each sample had at least two independent biological repeats. An asterisk (*) indicates a significant difference $(P<0.05)$.
Here, we demonstrate that TaTrxhl was also rapidly induced in wheat following pathogen inoculation. As a further step in defining the defense signaling processes associated with P. striiformis f. sp. tritici-based immune signaling in wheat, we leveraged one of the key activities of Trxs to define the breadth and specificity of the mechanism or mechanisms that underpin their role in immune signaling - namely, their role in buffering host tolerance to oxidative stress. The HR cell death is a local defense response induced, in large part, by the production and accumulation of reactive oxygen (Coll et al. 2011). As a function of the regulatory processes, associated ROS production in rice, for example, is dependent upon the activity of $\mathrm{OsPHO}_{2}$, whose activity is regulated in a redox-dependent manner by Trxs (Cao et al. 2014). To determine if TaTrxhl functions in a ROS-based defense mechanism following $P$. striiformis f. sp. tritici infection, we show that silencing of TaTrxh1 resulted in a significant reduction in $P$. striiformis f. sp. tritici-induced ROS production, with a concomitant increase in the expression of TaCAT and TaSOD, which are required for ROS scavenging. Conversely, overexpression of TaTrxh1 in tobacco ( $N$. benthamiana) leaves led to the induction of a localized cell-death response, demonstrating that TaTrxh1 is involved in ROS production and accumulation, as well as the activation of PCD signaling in response to pathogen infection.

SA-based signaling plays a critical role in plant immune activation in response to biotrophic pathogens. SA biosynthesis in plants occurs through two pathways, the phenylalanine pathway and the ICS1 pathway. And the expression of over $98 \%$ of SA-regulated genes is dependent on NPR1 (Qi et al. 2018). As an indicator of this dependency, it has been reported that $P R$ genes are induced by SA, with $P R l$ being a critical marker for the activation of this pathway (Fu and Dong 2013). In recent years, emerging data has demonstrated a role for Trxs in the local and systemic immune signaling processes under the control of SA (Mata-Pérez and Spoel 2019). For example, work by Tada et al. (2008) suggests that AtTrxh5 promotes SAinduced immune gene expression by regulating NPR 1 conformational status; this is achieved by the reduction of NPR1 disulfide bonds, leading to a release of NPR1 monomers for translocation into the nucleus. Subsequent work showed that this activity further correlated with the localization of AtTrxh5, which was observed in both the cytoplasm and nucleus, an observation consistent with the movement of NPR1 (Kneeshaw et al. 2014). In wheat defense signaling following $P$. striiformis f. sp. tritici infection, previous work described a role for SA (Liu et al. 2019). Herein, the observation of an increase in TaTrxhl mRNA accumulation following SA treatment, supports the hypothesis that TaTrxhl is involved in SA signaling following $P$. striiformis f. sp. tritici infection. Indeed, our data demonstrate that during the interaction between TaTrxhl-silenced plants and $P$. striiformis f. sp. tritici, TaICS1, TaPAL, TaNPR1, TaPR1, and TaPR3 mRNAs were significantly reduced following $P$. striiformis $\mathrm{f}$. sp. tritici infection, while SA accumulation was reduced following $P$. striiformis f. sp. tritici inoculation. These data suggest that the function of TaTrxh1 in wheat resistance to $P$. striiformis $\mathrm{f}$. $\mathrm{sp}$. tritici may depend upon the generation of the SA signal.

The ability to both recognize and reduce target proteins underpins the primary function of Trxs in the regulation of the cellular redox environment. Based on this, role, it is reasonable to assume that the interaction between Trx and one or more target proteins likely occurs at the Cys-rich disulfide bridge of the oxidized target protein. Following this initial interaction, the subsequent formation of a mixed disulfide bridge results in the release of the reduced protein, and the generation of an oxidized Trx (Mata-Pérez and Spoel 2019). In this study, we 
screened CP1 for its ability to interact with TaTrxh1, using a yeast two-hybrid-based assay and an in-planta BiFC approach. Through this, we demonstrate that TaTrxh1 interacted with TaCP1. This is significant, as TaCP1 is classified as a member of the RD19A-like subfamily of PLCPs, with the closest homology to Arabidopsis RD19C, previously demonstrated to function in the regulation of processes associated with disulfide bridge formation (Richau et al. 2012). In support of observations

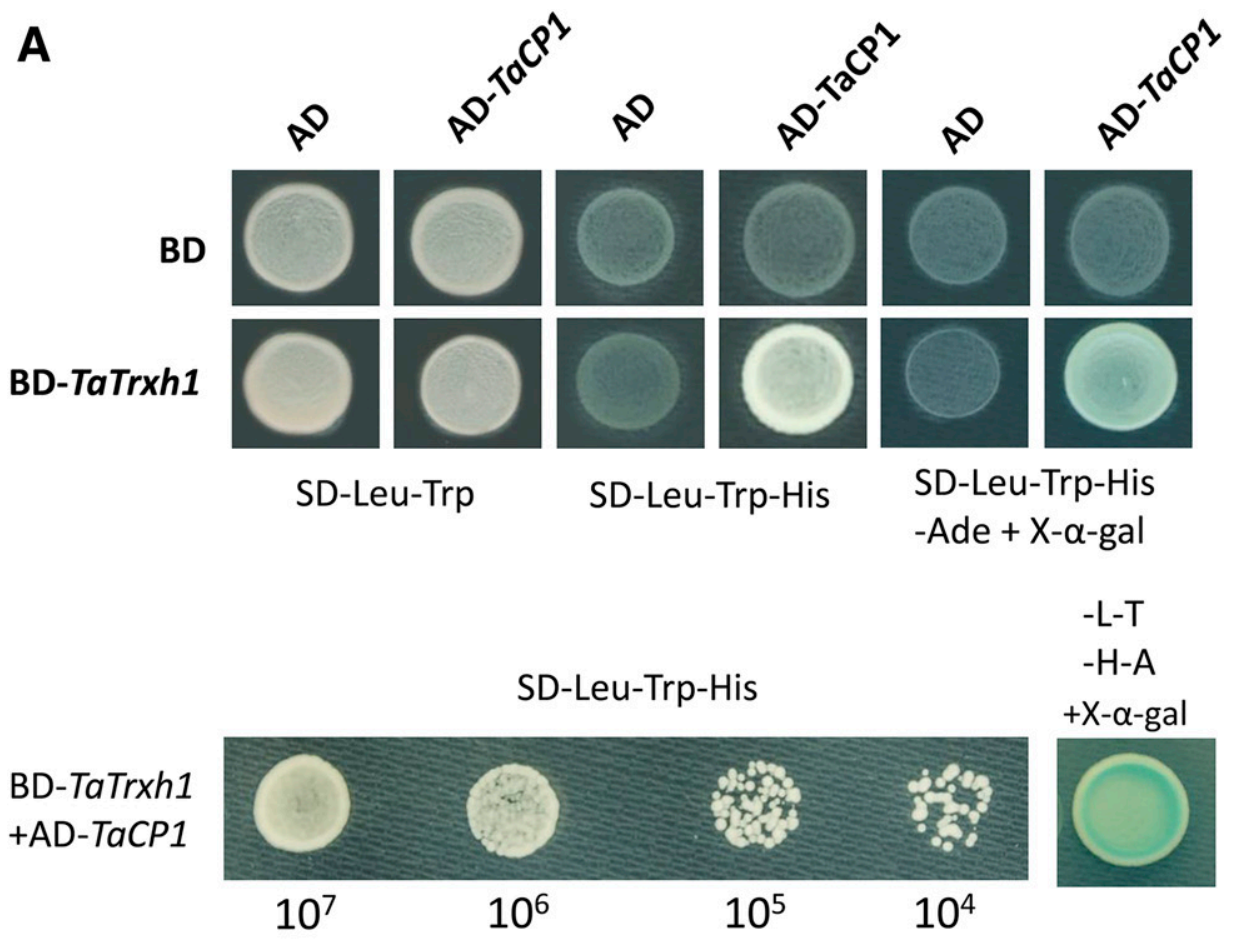

B

YFP
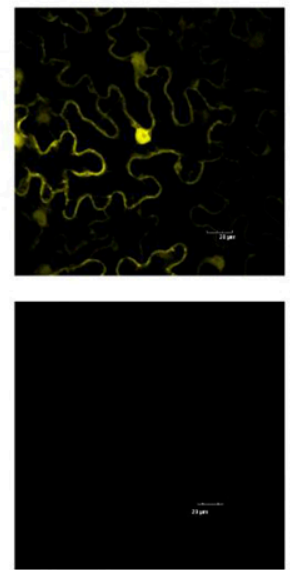

TaTrxh1-cYFP

+ nYFP

CYFP

+ TaCP1-nYFP

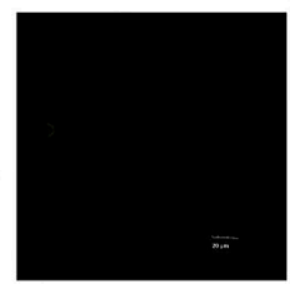

Brightfield
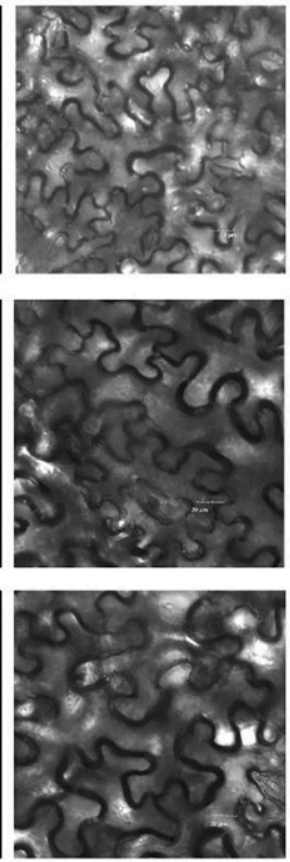

Merge
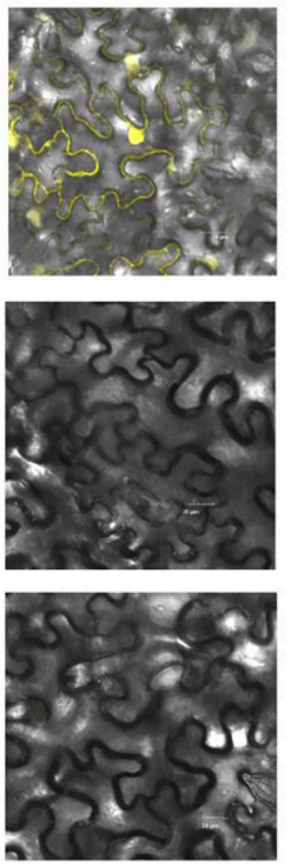

Fig. 6. TaTrxh1 interacts with TaCP1. A, Initial validation of TaTrxh1-TaCP1 interactions via the yeast two-hybrid system. Yeast strain Y2HGold containing TaTrxh1 and TaCP1 were grown SD-Leu-Trp-His and SD-Trp-Leu-His-Ade selective medium and were stained with X- $\alpha$-Gal solution. The negative control groups had no significant strain growth or color reaction. B, Bimolecular fluorescence complementation showed that TaTrxh1 interacts with TaCP1 in leaf cells of Nicotiana benthamiana. TaTrxh1 and TaCP1 were coexpressed in $N$. benthamiana by agroinfiltration. Yellow fluorescence signals were observed at 48 to 72 $\mathrm{h}$ postinoculation $(\mathrm{bars}=20 \mu \mathrm{m})$. 

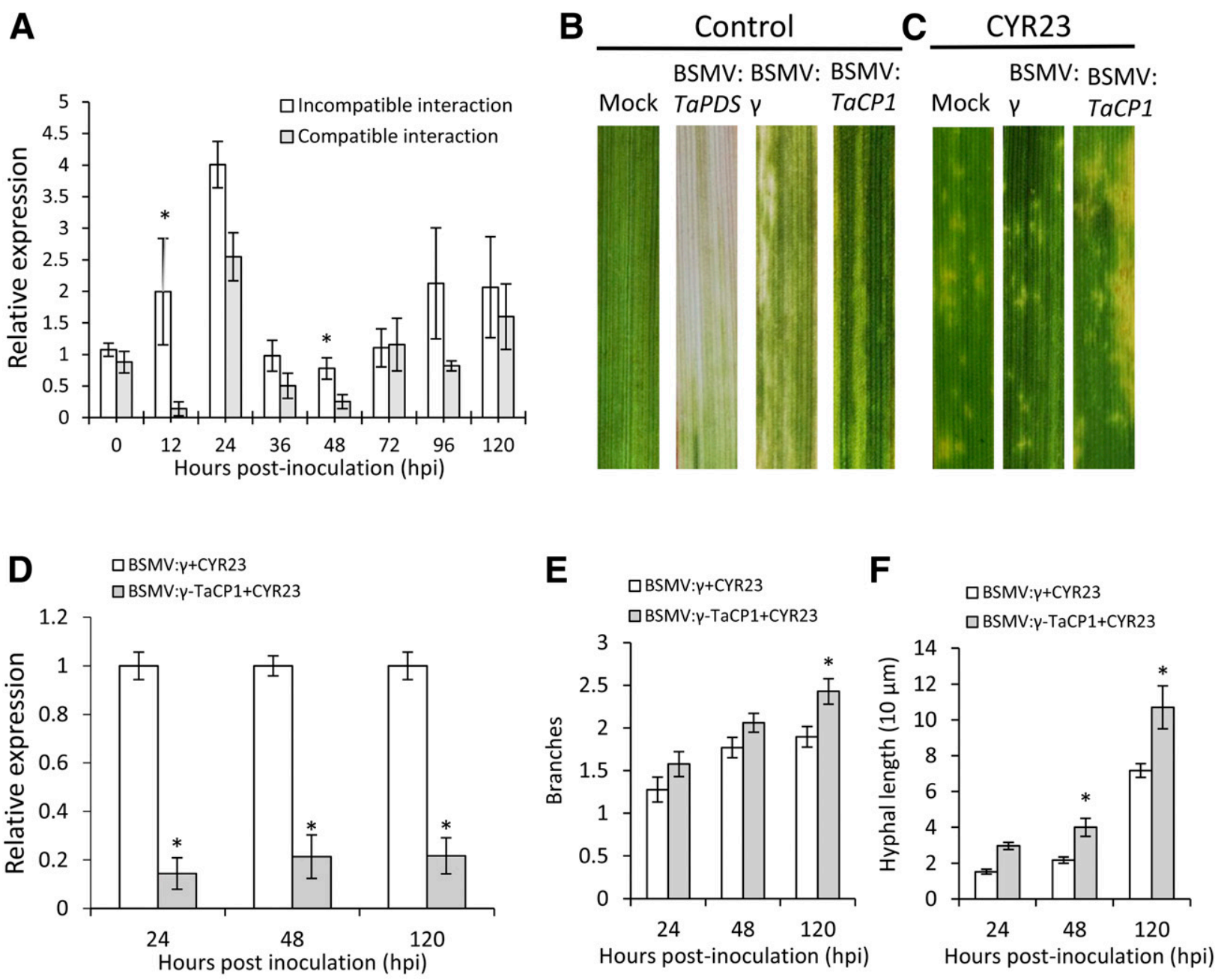

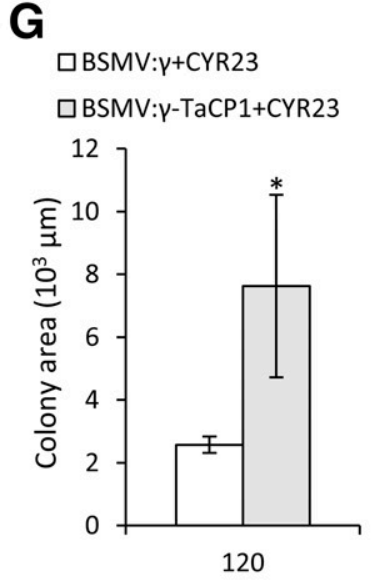

Hours post-inoculation (hpi)
H

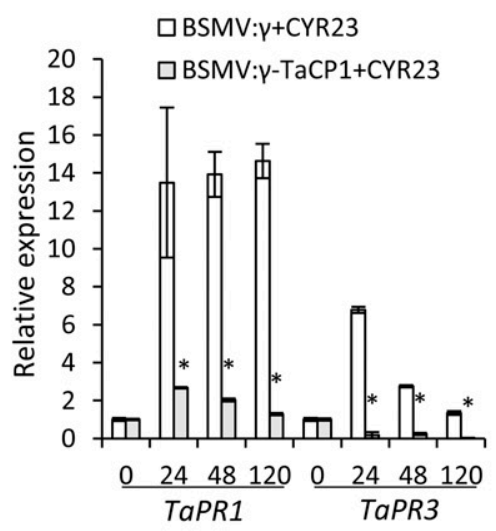

Hours post-inoculation (hpi)
I

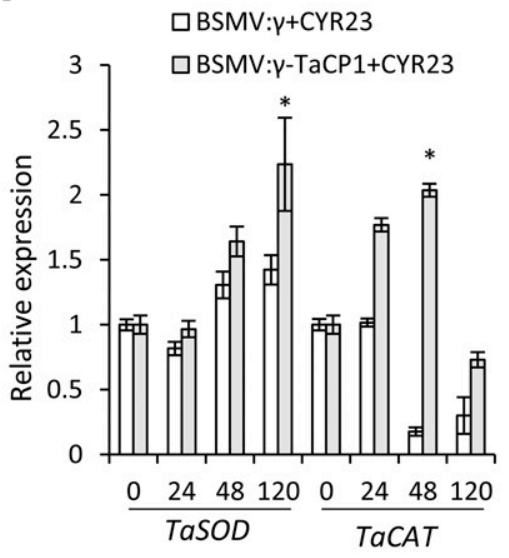

Hours post-inoculation (hpi)

Fig. 7. TaCP1 plays a positive role in wheat resistance to Puccinia striiformis $\mathrm{f}$. sp. tritici. A, Transcriptional level of TaCP1 as a function of $P$. striiformis $\mathrm{f}$. sp. tritici infection. Asterisks (*) indicate significant difference $(P<0.05)$ between $P$. striiformis f. sp. tritici races CYR23- (incompatible interaction) and CYR31 (compatible interaction)-infected Suwon 11 at the same timepoint, using Student's $t$ test. B, Barley stripe mosaic virus with a specific fragment of TaCP1 was pretreated on the second leaves of wheat. After 10 days, photobleaching and mild chlorotic mosaic symptoms were observed on the fourth leaves. C, Functional characterization of TaCP1 using barley stripe mosaic virus (BSMV)-induced gene silencing. Urediospores of CYR23 were inoculated onto silenced plants and were evaluated at 15 days postinoculation. Representative leaves are shown. D, Effective silencing of TaCP1. E, Number of hyphal branches, F, hyphal length, and $\mathbf{G}$, colony area were all recorded from 50 infection sites. $\mathbf{H}$ and $\mathbf{I}$, Transcript analysis of defense-related genes after $P$. striiformis $\mathbf{f}$. sp. tritici inoculation in silenced leaves. TaPRI = pathogenesis-related protein (AJ007348.1), TaPR3 = chitinase 3 (AB029936.1), TaSOD = superoxide dismutase $($ AK453889.1), and $T a C A T=$ catalase $(\mathrm{AF} 475100.1)$. The $2^{-\Delta \Lambda \mathrm{Ct}}$ method was used and each sample had three independent biological repeats. Asterisks $(*)$ indicate significant difference $(P<0.05)$, using Student's $t$ test. TaEF-1 $a$ was used as a control. 
presented herein, PLCPs are crucial enzymes functioning in the regulation of PCD and pathogen resistance. For example, in Arabidopsis, AtRD19 has been demonstrated as a required signaling protein associated with PopP2-mediated plant immunity (Bernoux et al. 2008). Among all identified RD19 homolog proteins in wheat, $\mathrm{TaCP} 1$ was the only one confirmed to have a function associated with resistance signaling in response to $P$. striiformis f. $\mathrm{sp}$. tritici. Indeed, our data demonstrate that TaTrxh1 specifically interacts with TaCP1 in planta and that this interaction positively regulates cell-death elicitation following P. striiformis f. sp. tritici infection (Fig. 8).

In summary, we identified an $h$-type Trx, TaTrxh1, as a positive regulator of immunity in wheat. Unlike previously reported Trxs, TaTrxh1 can directly induce the activation of cell-death signaling, a function that likely underpins the downstream activation of disease resistance through its interaction with TaCP1. In total, the data presented herein provide direct evidence that TaTrxh1 functions in an SA-dependent manner to regulate immune signaling, and moreover, through its interaction with TaCP1, it plays a key, indispensable, role in the activation of robust immunity to the stripe rust pathogen $P$. striiformis f. sp. tritici. These data lay a solid foundation from which future mechanistic studies will emerge to describe the role of cellular redox and PCD in response to fungal pathogenesis in plants.

\section{MATERIALS AND METHODS}

Plant and pathogen material, treatment, and growth.

The wheat cultivar Su11 plants that carried the $\mathrm{YrSu}$ resistance gene were grown at $25^{\circ} \mathrm{C}$, with a day and night cycle of 16 and $8 \mathrm{~h}$, respectively, and were used for all experiments described here. P. striiformis f. sp. tritici race CYR23 (avirulent) was used as an incompatible pathogen, while race CYR31 (virulent) was used as a compatible interaction (Cao et al. 2003).

The urediospores of $P$. striiformis f. sp. tritici (CYR23 and CYR31, individually) were grown on wheat cv. Mingxian169 leaves. The inoculated plants were placed in a dark incubator with a temperature of $10^{\circ} \mathrm{C}$ and a relative humidity of $90 \%$ for $24 \mathrm{~h}$. After $24 \mathrm{~h}$, inoculated plants were moved to an incubator at $16^{\circ} \mathrm{C}$, under a day and night cycle of 16 and $8 \mathrm{~h}$, respectively. After $12 \mathrm{dpi}$, the fresh urediospores of stripe rust were collected (Kang and Li 1984).

To evaluate the expression levels of TaTrxhl and TaCP1 in response to $P$. striiformis $\mathrm{f}$. sp. tritici infection, Su11 seedlings were inoculated with urediospores of CYR23 and CYR31, and leaves were harvested at $0,12,24,36,48,72,96$, and $120 \mathrm{hpi}$. For hormone treatment, $100 \mu \mathrm{M}$ MeJA and $2 \mu \mathrm{M}$ SA were sprayed on Su11 leaves, and samples were taken at 0, 0.5, 3, 6, 12, and $24 \mathrm{hpt}$. All leaves were treated with distilled water as a control, and each treatment was repeated three times (Zhang et al. 2017).

Tobacco ( $N$. benthamiana) was grown at $25^{\circ} \mathrm{C}$ under a day and night cycle of 14 and $10 \mathrm{~h}$, respectively. After 4 to 6 weeks, at the six-leaf stage, leaves were inoculated with A. tumefaciens GV3101 containing one of several PVX106-derived vectors for protein overexpression.

Escherichia coli DH5 $\alpha$ was grown in Luria-Bertani (LB) medium at $37^{\circ} \mathrm{C}$. A. tumefaciens GV3101 was grown in LB media at $28^{\circ} \mathrm{C}$. Saccharomyces cerevisiae $\mathrm{Y} 2 \mathrm{H}$ Gold was grown on YPDA (1\% yeast extract, $2 \%$ peptone, $2 \%$ dextrose, $0.02 \%$ adenine) medium at $30^{\circ} \mathrm{C}$.

\section{DNA cloning and sequence analysis.}

Full-length DNA sequences with 347 bp were isolated from wheat cv. Su11 by homology-based cloning, using wheat Trxh as a template (GenBank accession number AY072771.1). The open reading frame (ORF) sequence of TaTrxh1 was analyzed using the National Center for Biotechnology Information (NCBI) ORF Finder and URGI BLASTN. The protein sequence of TaTrxhl was analyzed using Protparam. Multiple sequence alignments were performed using DNAMAN6.0. A phylogenetic tree was constructed with the MEGA 6.0 software (Sun et al. 2019).

\section{RT-qPCR analysis.}

Total RNA was isolated using Biozol total RNA extraction kit (Bioflux). After evaluation of quality, $2 \mu \mathrm{g}$ of RNA from each sample was subjected to first strand cDNA synthesis using FastKing cDNA Kit (with gDNase) (Tiangen). Primer Premier 6.0 software was used to design the DNA primers for RT-qPCR (Supplementary Table S1). DNA primers were evaluated using the NCBI Primer-BLAST tool to determine target site specificity. The total reaction system and RT-qPCR protocol was as recommended for the UltraSYBR mixture system (KangWei). After the reaction, the $2^{-\Delta \Delta \mathrm{Ct}}$ method was used for quantitation, with the TaEF-la gene (GenBank accession number Q0303033) as control gene. After repeating the reaction in triplicate, the SPSS 23.0 software was used to determine statistical significance $(P<$ 0.05) (Livak and Schmittgen 2001).

\section{Pst infection}

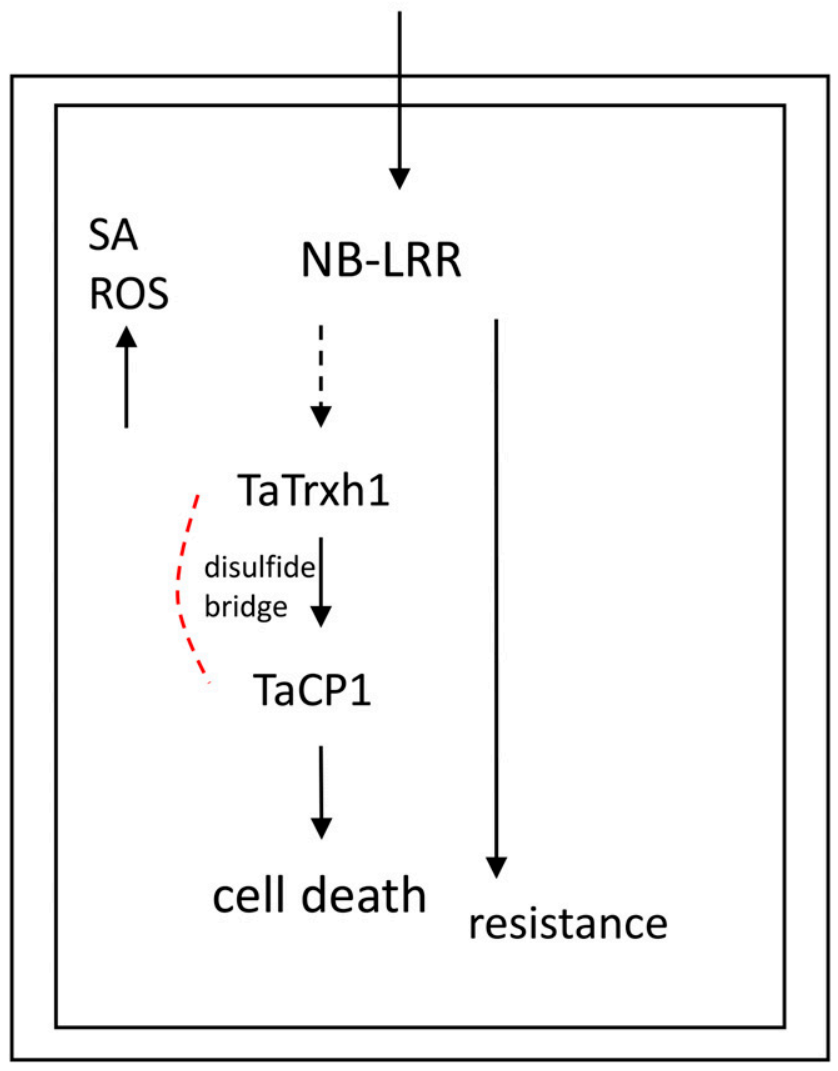

Fig. 8. A hypothetical model of the function of TaTrxh1 that positively regulates cell death at the site of Puccinia striiformis f. sp. tritici (Pst) infection. In plants, TaTrxh1 directly associates with TaCP1, which may regulate the redox reaction by reduction of the disulfide bond and positively regulates cell death mediated by nucleo binding-leucine-rich repeat (NBLRR) recognition of the invading $P$. striiformis $\mathrm{f}$. sp. tritici. In the process, the accumulation of reactive oxygen species (ROS) is induced and TaTrxh1 and TaCP1 can regulate TaSOD and TaCAT to scavenge ROS. In addition, TaTrxh1 may regulate wheat resistance to $P$. striiformis $\mathrm{f}$. sp. tritici through salicylic acid (SA) signal. 


\section{Overexpression of TaTrxh1 protein in $N$. benthamiana.}

The full ORF of TaTrxh1 was cloned into the PVX106 vector via SalI digestion and ligation. After vector construction, PVX106: TaTrxh1 was transformed into A. tumefaciens GV3101. Cultures of $A$. tumefaciens were centrifuged and the resultant bacterial pellets were resuspended in infiltration buffer $(10 \mathrm{mM}$ morpholineethane sulfonic acid, $\mathrm{pH} 5.6$ ) to a final optical density at $600 \mathrm{~nm}$ of 0.6. With A. tumefaciens infecting $N$. benthamiana, the Bax site was infiltrated with Bax at 0 hpi and was the positive control for PVX106:TaTrxh1, PVX106 vector was the blank control, and infiltration buffer was the negative control. The PVX106+ Bax site was infiltrated with PVX106 earlier and challenged with Bax at $24 \mathrm{hpi}$, which was the positive control for PVX106:TaTrxh1+Bax to determine whether TaTrxhl could suppress PCD. The cell-death symptoms caused by Bax were monitored at $5 \mathrm{dpi}$, which were then decolorized with $100 \%$ alcohol and were photographed. The experiment was repeated at least two times.

\section{BSMV-mediated gene silencing.}

The special segment of TaTrxhl (Supplementary Fig. S1B) was constructed into BSMV: $\gamma$ vector with NotI and PacI restriction enzyme for gene silencing. The BSMV RNAs were transcribed in vitro from linearized plasmids BSMV: $\gamma$-gene, $\gamma$-TaPDS (phytoene desaturase), BSMV: $\gamma, \mathrm{BSMV}: \alpha, \mathrm{BSMV}: \beta$ according to manufacturer instructions (RiboMAX Large Scale RNA Production Systems-T7). The BSMV RNAs ( $\alpha, \beta$, and $\gamma / \gamma$ genes) were mixed with FES buffer and were inoculated on the second leaf of two-leaf Su11. The plants were then put in a dark incubator with temperature of $23 \pm 1{ }^{\circ} \mathrm{C}$ and humidity of $90 \%$ for $24 \mathrm{~h}$, with an adjusted day and night cycle of 16 and $8 \mathrm{~h}$, respectively (Wang et al. 2012b). Ten days after virus inoculation, fresh CYR23 and CYR31 urediniospores were inoculated onto the fourth leaf, and the leaves were sampled at $0,24,48$, and 120 hpi for isolating total RNA, were sampled at 24, 48, and $120 \mathrm{hpi}$ for histological observation, and were sampled at 18 and $24 \mathrm{hpi}$ for quantification of SA and JA. At 15 dpi with $P$. striiformis f. sp. tritici, phenotypes were observed and photographed. The experiment was repeated three times.

We measured the expression of TaTrxhl and TaCPl in silenced plants to determine silencing efficiency. In addition, to analyze effects of gene expression on plant resistance at the molecular level, we designed the RT-qPCR primers to TaPRl (pathogenesis-related protein), TaPR3 (chitinase 3), TaSOD, TaCAT, TaICS1, TaPAL, and TaNPR1 and then measured the expression of these genes.

\section{Histological observation of $\boldsymbol{P}$. striiformis f. sp. tritici development in gene-silenced plants.}

The samples at each timepoint $(0,24,48$, and $120 \mathrm{hpi})$ were stained using $1 \mathrm{mg}$ of DAB (3,3-diaminobenzidine) per mililiter (Amresco) to detect the production and accumulation of $\mathrm{H}_{2} \mathrm{O}_{2}$. Following staining, leaves were decolorized using $100 \%$ ethanol and were stained with WGA AF488 to observe P. striiformis f. sp. tritici development; this included evaluating the number of hyphal branches, hyphal length, and colony area. Using a NIKON-80i microscope, 50 infection points were randomly inspected for each treatment. The development of a substomatal vesicle was regarded as successful $P$. striiformis f. sp. tritici infection (Ayliffe et al. 2011). The proportions of $\mathrm{H}_{2} \mathrm{O}_{2}$ areas, number of hyphal branches, hyphal length, and colony area were observed by microscopy and were calculated using DP-BSW software (Olympus LifeScience). The SPSS (ver. 23.0) software was used to determine statistical significance $(P<0.05)$.

\section{Quantification of SA and JA by liquid chromatography-tandem mass spectrometry (LC-MS) in TaTrxh1-silenced plants.}

After TaTrxh1 silencing, the silenced and control plants were inoculated with P. striiformis f. sp. tritici CYR23. At 18 and
$24 \mathrm{hpi}, 500 \mathrm{mg}$ of leaf tissue was sampled, was frozen in liquid nitrogen, and was stored at $-80^{\circ} \mathrm{C}$. Frozen samples were ground with a mortar and pestle under liquid nitrogen and $2 \mathrm{ml}$ of methanol/water/acetic acid (90:9:1, vol/vol/vol) and were centrifuged for $1 \mathrm{~min}$ at $10,000 \mathrm{rpm}$. The extraction was repeated twice with $400 \mu \mathrm{l}$ to collect the supernatant. The resultant was then dried under nitrogen, and $200 \mu \mathrm{l}$ of methanol was added to resuspend the dried sample. After filtering through a $0.2 \mathrm{~mm}$ polytetrafluoroethylene membrane, the samples were detected by LC-MS, and SA and JA were quantified based on standard curves (Zhang et al. 2017).

\section{Yeast two-hybrid and BiFC analysis.}

The full ORF of TaTrxhl was cloned into pGBKT7 (BD) vector. Then, the plasmid was transferred into Y2H Gold following the Yeast Protocols Handbook (Takara). The cDNA library constructed with wheat infected by $P$. striiformis f. sp. tritici was transformed into the yeast containing pGBKT7TaTrxh1, to screen candidate interaction proteins. After selection on SD-Leu-Trp-His and SD-Leu-Trp-His+X- $\alpha$-Gal medium, PCR was carried out on the positive clones and the products of PCR reaction were sent to Sangon Biotech to obtain DNA sequences. The results of those DNA sequences were analyzed and compared using the NCBI BLAST tool.

The candidate protein sequence of $T a C P l$ was cloned into pGADT7 (AD), and the pairs of recombinant plasmids of pGADT7-TaCP1 and pGBKT7-TaTrxhl were cotransformed into Y2H Gold. Meanwhile, the negative controls (pGBKT7+pGADT7, pGBKT7-TaTrxh1+pGADT7, pGBKT7+pGADT7-TaCP1) were also cotransformed into $\mathrm{Y} 2 \mathrm{H}$ Gold.

To verify the screening results of yeast two-hybrid, a published BiFC procedure was used (Waadt et al. 2008). The ORF sequence TaTrxhl and TaCP1 were constructed on PSPYCE and pSPYNE, respectively. The constructed vector was transformed into GV3101 to coexpress protein in 4-week-old $N$. benthamiana including pSPYCE-TaTrxh1+pSPYNE-TaCP1, pSPYCE+pSPYNE-TaCP1, and pSPYCE-TaTrxh1+pSPYNE. From 48 to $72 \mathrm{~h}$ after coagroinfection, yellow fluorescence was observed using confocal laser scanning microscopy (Olympus FV1000MPE) with the FV10-ASW 3.1 software suite. The assay was repeated in triplicate.

\section{AUTHOR-RECOMMENDED INTERNET RESOURCES}

NCBI ORF Finder: https://www.ncbi.nlm.nih.gov/orffinder Protparam: https://web.expasy.org/cgi-bin/protparain/protParam URGI BLASTN: https://urgi.versailles.inra.fr/blast

\section{LITERATURE CITED}

Arsova, B., Hoja, U., Wimmelbacher, M., Greiner, E., Ustün, S., Melzer, M., Petersen, K., Lein, W., and Börnke, F. 2010. Plastidial thioredoxin z interacts with two fructokinase-like proteins in a thiol-dependent manner: Evidence for an essential role in chloroplast development in Arabidopsis and Nicotiana benthamiana. Plant Cell 22:1498-1515.

Ayliffe, M., Devilla, R., Mago, R., White, R., Talbot, M., Pryor, A., and Leung, H. 2011. Nonhost resistance of rice to rust pathogens. Mol. PlantMicrobe Interact. 24:1143-1155.

Bashandy, T., Guilleminot, J., Vernoux, T., Caparros-Ruiz, D., Ljung, K., Meyer, Y., and Reichheld, J. P. 2010. Interplay between the NADPlinked thioredoxin and glutathione systems in Arabidopsis auxin signaling. Plant Cell 22:376-391.

Bennypaul, H. S., Mutti, J. S., Rustgi, S., Kumar, N., Okubara, P. A., and Gill, K. S. 2012. Virus-induced gene silencing (VIGS) of genes expressed in root, leaf, and meiotic tissues of wheat. Funct. Integr. Genomics 12:143-156.

Bernoux, M., Timmers, T., Jauneau, A., Brière, C., de Wit, P. J. G. M., Marco, Y., and Deslandes, L. 2008. RD19, an Arabidopsis cysteine protease required for RRS1-R-mediated resistance, is relocalized to the nucleus by the Ralstonia solanacearum PopP2 effector. Plant Cell 20: 2252-2264. 
Cao, Y., Yan, Y., Zhang, F., Wang, H. D., Gu, M., Wu, X. N., Sun, S. B., and $\mathrm{Xu}, \mathrm{G}$. H. 2014. Fine characterization of $\mathrm{OsPHO} 2$ knockout mutants reveals its key role in Pi utilization in rice. J. Plant Physiol. 171:340-348.

Cao, Z., Jing, J., Wang, M., Shang, H., and Li, Z. 2003. Relation analysis of stripe rust resistance gene in wheat important cultivar suwon 11, suwon 92 and hybrid 46. Acta Bot. Boreali-occidentalia Sin. 23:64-68.

Chen, W., Wellings, C., Chen, X., Kang, Z., and Liu, T. 2014. Wheat stripe (yellow) rust caused by Puccinia striiformis f. sp. tritici. Mol. Plant Pathol. 15:433-446.

Coll, N. S., Epple, P., and Dangl, J. L. 2011. Programmed cell death in the plant immune system. Cell Death Differ. 18:1247-1256.

Dodds, P. N., and Rathjen, J. P. 2010. Plant immunity: Towards an integrated view of plant-pathogen interactions. Nat. Rev. Genet. 11: 539-548.

Duan, Y. H., Guo, J., Ding, K., Wang, S. J., Zhang, H., Dai, X. W., Chen, Y. Y., Govers, F., Huang, L. L., and Kang, Z. S. 2011. Characterization of a wheat HSP70 gene and its expression in response to stripe rust infection and abiotic stresses. Mol. Biol. Rep. 38:301-307.

Eichmann, R., and Hückelhoven, R. 2008. Accommodation of powdery mildew fungi in intact plant cells. J. Plant Physiol. 165:5-18.

Fu, Z. Q., and Dong, X. 2013. Systemic acquired resistance: Turning local infection into global defense. Annu. Rev. Plant Biol. 64:839-863.

Gelhaye, E., Rouhier, N., Navrot, N., and Jacquot, J. P. 2005. The plant thioredoxin system. Cell. Mol. Life Sci. 62:24-35.

Jacquot, J.-P., Gelhaye, E., Rouhier, N., Corbier, C., Didierjean, C., and Aubry, A. 2002. Thioredoxins and related proteins in photosynthetic organisms: Molecular basis for thiol dependent regulation. Biochem. Pharmacol. 64:1065-1069.

Jones, J. D., and Dangl, J. L. 2006. The plant immune system. Nature 444: 323-329.

Kang, Z., and Li, Z. 1984. Discovery of a normal T. type new pathogenic strain to Lovrin10. Acta Cllegii Septentrionali Occidentali Agric. 4: 18-28.

Kneeshaw, S., Gelineau, S., Tada, Y., Loake, G. J., and Spoel, S. H. 2014. Selective protein denitrosylation activity of thioredoxin- $h 5$ modulates plant immunity. Mol. Cell 56:153-162.

Lacomme, C., and Santa Cruz, S. 1999. Bax-induced cell death in tobacco is similar to the hypersensitive response. Proc. Natl. Acad. Sci. U.S.A. 96:7956-7961.

Laloi, C., Mestres-Ortega, D., Marco, Y., Meyer, Y., and Reichheld, J. P. 2004. The Arabidopsis cytosolic thioredoxin $h 5$ gene induction by oxidative stress and its W-box-mediated response to pathogen elicitor. Plant Physiol. 134:1006-1016.

Liu, P., Guo, J., Zhang, R., Zhao, J., Liu, C., Qi, T., Duan, Y., Kang, Z., and Guo, J. 2019. TaCIPK10 interacts with and phosphorylates TaNH2 to activate wheat defense responses to stripe rust. Plant Biotechnol. J. 17: 956-968.

Livak, K. J., and Schmittgen, T. D. 2001. Analysis of relative gene expression data using real-time quantitative PCR and the $2^{-\Delta \Delta \mathrm{Ct}}$ method. Methods 25:402-408

Macho, A. P., and Zipfel, C. 2015. Targeting of plant pattern recognition receptor-triggered immunity by bacterial type-III secretion system effectors. Curr. Opin. Microbiol. 23:14-22.

Marchal, C., Delorme-Hinoux, V., Bariat, L., Siala, W., Belin, C., SaezVasquez, J., Riondet, C., and Reichheld, J. P. 2014. NTR/NRX define a new thioredoxin system in the nucleus of Arabidopsis thaliana cells. Mol. Plant 7:30-44

Mata-Pérez, C., and Spoel, S. H. 2019. Thioredoxin-mediated redox signalling in plant immunity. Plant Sci. 279:27-33.

Meyer, Y., Belin, C., Delorme-Hinoux, V., Reichheld, J. P., and Riondet, C. 2012. Thioredoxin and glutaredoxin systems in plants: Molecular mechanisms, crosstalks, and functional significance. Antioxid. Redox Signal. 17:1124-1160.

Meyer, Y., Buchanan, B. B., Vignols, F., and Reichheld, J. P. 2009. Thioredoxins and glutaredoxins: Unifying elements in redox biology. Annu. Rev. Genet. 43:335-367.

Meyer, Y., Siala, W., Bashandy, T., Riondet, C., Vignols, F., and Reichheld, J. P. 2008. Glutaredoxins and thioredoxins in plants. Biochim. Biophys. Acta 1783:589-600.

Meyer, Y., Vignols, F., and Reichheld, J. P. 2002. Classification of plant thioredoxins by sequence similarity and intron position. Methods Enzymol. 347:394-402.

Mukaihara, T., Hatanaka, T., Nakano, M., and Oda, K. 2016. Ralstonia solanacearum type III effector RipAY is a glutathione-degrading enzyme that is activated by plant cytosolic thioredoxins and suppresses plant immunity. MBio 7:e00359-16.

Park, S. K., Jung, Y. J., Lee, J. R., Lee, Y. M., Jang, H. H., Lee, S. S., Park, J. H., Kim, S. Y., Moon, J. C., Lee, S. Y., Chae, H. B., Shin, M. R., Jung,
J. H., Kim, M. G., Kim, W. Y., Yun, D. J., Lee, K. O., and Lee, S. Y. 2009. Heat-shock and redox-dependent functional switching of an $h$-type Arabidopsis thioredoxin from a disulfide reductase to a molecular chaperone. Plant Physiol. 150:552-561.

Qi, G., Chen, J., Chang, M., Chen, H., Hall, K., Korin, J., Liu, F., Wang, D., and $\mathrm{Fu}, \mathrm{Z}$. Q. 2018. Pandemonium breaks out: Disruption of salicylic acid-mediated defense by plant pathogens. Mol. Plant 11:1427-1439.

Reichheld, J.-P., Mestres-Ortega, D., Laloi, C., and Meyer, Y. 2002. The multigenic family of thioredoxin $\mathrm{h}$ in Arabidopsis thaliana: Specific expression and stress response. Plant Physiol. Biochem. 40:685-690.

Reichheld, J. P., Riondet, C., Delorme, V., Vignols, F., and Meyer, Y. 2010. Thioredoxins and glutaredoxins in development. Plant Sci. 178:420-423.

Richau, K. H., Kaschani, F., Verdoes, M., Pansuriya, T. C., Niessen, S., Stüber, K., Colby, T., Overkleeft, H. S., Bogyo, M., and Van der Hoorn, R. A. 2012. Subclassification and biochemical analysis of plant papainlike cysteine proteases displays subfamily-specific characteristics. Plant Physiol. 158:1583-1599.

Rivas, S., Rougon-Cardoso, A., Smoker, M., Schauser, L., Yoshioka, H., and Jones, J. D. 2004. CITRX thioredoxin interacts with the tomato Cf-9 resistance protein and negatively regulates defence. EMBO J. 23: 2156-2165.

Sahrawy, M., Hecht, V., Lopez-Jaramillo, J., Chueca, A., Chartier, Y., and Meyer, Y. 1996. Intron position as an evolutionary marker of thioredoxins and thioredoxin domains. J. Mol. Evol. 42:422-431.

Singh, R. P., Singh, P. K., Rutkoski, J., Hodson, D. P., He, X., Jørgensen, L. N., Hovmøller, M. S., and Huerta-Espino, J. 2016. Disease impact on wheat yield potential and prospects of genetic control. Annu. Rev. Phytopathol. 54:303-322.

Spector, A., Yan, G. Z., Huang, R. R., McDermott, M. J., Gascoyne, P. R. C., and Pigiet, V. 1988. The effect of $\mathrm{H}_{2} \mathrm{O}_{2}$ upon thioredoxinenriched lens epithelial cells. J. Biol. Chem. 263:4984-4990.

Sun, G., Feng, C., Guo, J., Zhang, A., Xu, Y., Wang, Y., Day, B., and Ma, Q. 2019. The tomato Arp $2 / 3$ complex is required for resistance to the powdery mildew fungus Oidium neolycopersici. Plant Cell Environ. 42: 2664-2680.

Sun, L., Ren, H., Liu, R., Li, B., Wu, T., Sun, F., Liu, H., Wang, X., and Dong, H. 2010. An h-type thioredoxin functions in tobacco defense responses to two species of viruses and an abiotic oxidative stress. Mol. Plant-Microbe Interact. 23:1470-1485.

Tada, Y., Spoel, S. H., Pajerowska-Mukhtar, K., Mou, Z., Song, J., Wang, C., Zuo, J., and Dong, X. 2008. Plant immunity requires conformational changes [corrected] of NPR1 via S-nitrosylation and thioredoxins. Science 321:952-956.

Thormählen, I., Zupok, A., Rescher, J., Leger, J., Weissenberger, S., Groysman, J., Orwat, A., Chatel-Innocenti, G., Issakidis-Bourguet, E., Armbruster, U., and Geigenberger, P. 2017. Thioredoxins play a crucial role in dynamic acclimation of photosynthesis in fluctuating light. Mol. Plant 10:168-182.

Van Hautegem, T., Waters, A. J., Goodrich, J., and Nowack, M. K. 2015. Only in dying, life: Programmed cell death during plant development. Trends Plant Sci. 20:102-113.

Vieira Dos Santos, C., and Rey, P. 2006. Plant thioredoxins are key actors in the oxidative stress response. Trends Plant Sci. 11:329-334.

Waadt, R., Schmidt, L. K., Lohse, M., Hashimoto, K., Bock, R., and Kudla, J. 2008. Multicolor bimolecular fluorescence complementation reveals simultaneous formation of alternative CBL/CIPK complexes in planta. Plant J. 56:505-516.

Wang, F., Kong, W., Niu, Y., Ye, Y., Fan, S., Wang, Y., Chen, X., and Zhou, Q. 2017. StTrxF, a potato plastidic thioredoxin F-type protein gene, is involved in starch accumulation in transgenic Arabidopsis thaliana. Biotechnol. Biotechnol. Equip. 31:486-492.

Wang, X., Tang, C., Huang, X., Li, F., Chen, X., Zhang, G., Sun, Y., Han, D., and Kang, Z. 2012b. Wheat BAX inhibitor-1 contributes to wheat resistance to Puccinia striiformis. J. Exp. Bot. 63:4571-4584.

Wang, X., Wang, X., Deng, L., Chang, H., Dubcovsky, J., Feng, H., Han, Q., Huang, L., and Kang, Z. 2014. Wheat TaNPSN SNARE homologues are involved in vesicle-mediated resistance to stripe rust (Puccinia striiformis f. sp. tritici). J. Exp. Bot. 65:4807-4820.

Wang, X., Wang, X., Feng, H., Tang, C., Bai, P., Wei, G., Huang, L., and Kang, Z. 2012a. TaMCA4, a novel wheat metacaspase gene functions in programmed cell death induced by the fungal pathogen Puccinia striiformis f. sp. tritici. Mol. Plant-Microbe Interact. 25:755-764.

Xie, G., Kato, H., Sasaki, K., and Imai, R. 2009. A cold-induced thioredoxin $\mathrm{h}$ of rice, OsTrx23, negatively regulates kinase activities of OsMPK3 and OsMPK6 in vitro. FEBS Lett. 583:2734-2738.

Yi, S. Y., Choi, D., and Ryu, C.-M. 2007. Implication of a pepper $h$-type thioredoxin in type I- and II-nonhost resistance to Xanthomonas axonopodis. Plant Biotechnol. Rep. 1:117-123. 
Yun, B. W., Skelly, M. J., Yin, M., Yu, M., Mun, B. G., Lee, S. U., Hussain, A., Spoel, S. H., and Loake, G. J. 2016. Nitric oxide and Snitrosoglutathione function additively during plant immunity. New Phytol. 211:516-526.

Zhang, B., Hua, Y., Wang, J., Huo, Y., Shimono, M., Day, B., and Ma, Q. 2017. TaADF4, an actin-depolymerizing factor from wheat, is required for resistance to the stripe rust pathogen Puccinia striiformis $\mathrm{f}$. sp. tritici. Plant J. 89:1210-1224.

Zhang, C. J., Zhao, B. C., Ge, W. N., Zhang, Y. F., Song, Y., Sun, D. Y., and Guo, Y. 2011. An apoplastic h-type thioredoxin is involved in the stress response through regulation of the apoplastic reactive oxygen species in rice. Plant Physiol. 157:1884-1899.

Zhang, Y., Zhang, G., Xia, N., Wang, X., Huang, L., and Kang, Z. 2008. Cloning and characterization of a bZIP transcription factor gene in whea and its expression in response to stripe rust pathogen infection and abiotic stresses. Physiol. Mol. Plant Pathol. 73:88-94.

Zhou, C., Bian, M., Liao, H., Mao, Q., Li, R., Zhou, J., Wang, X., Li, S., Liang, C., Li, X., Huang, Y., and Yu, X. 2013. Identification and immunological characterization of thioredoxin transmembrane-related protein from Clonorchis sinensis. Parasitol. Res. 112:1729-1736. 